\title{
Path Planning and Control of an Autonomous Quadrotor Testbed in a Cluttered Environment
}

Trevor W. Caplinger

Follow this and additional works at: https://researchrepository.wvu.edu/etd

\section{Recommended Citation}

Caplinger, Trevor W., "Path Planning and Control of an Autonomous Quadrotor Testbed in a Cluttered Environment" (2015). Graduate Theses, Dissertations, and Problem Reports. 5313.

https://researchrepository.wvu.edu/etd/5313

This Thesis is protected by copyright and/or related rights. It has been brought to you by the The Research Repository @ WVU with permission from the rights-holder(s). You are free to use this Thesis in any way that is permitted by the copyright and related rights legislation that applies to your use. For other uses you must obtain permission from the rights-holder(s) directly, unless additional rights are indicated by a Creative Commons license in the record and/ or on the work itself. This Thesis has been accepted for inclusion in WVU Graduate Theses, Dissertations, and Problem Reports collection by an authorized administrator of The Research Repository @ WVU. For more information, please contact researchrepository@mail.wvu.edu. 


\title{
Path Planning and Control of an Autonomous Quadrotor Testbed in a Cluttered Environment
}

\author{
Trevor W. Caplinger
}

\author{
Thesis submitted to \\ the Benjamin M. Statler College of Engineering and Mineral Resources \\ at West Virginia University \\ in partial fulfillment of the requirements for the degree of \\ Master of Science in
Mechanical Engineering
}

Committee Members
Yu Gu, Ph.D., Chair
Jason Gross, Ph.D.
John Christian, Ph.D.
Powsiri Klinkhachorn, Ph.D.
Department of Mechanical and Aerospace Engineering

Morgantown, West Virginia

2015

Keywords: Quadrotor, Quadcopter, Path Planning, D*, PRM, Control Copyright 2015 Trevor Caplinger 


\title{
Abstract \\ Path Planning and Control of an Autonomous Quadrotor Testbed in a Cluttered Environment
}

\author{
Trevor W. Caplinger
}

A classical problem for robotic navigation is how to efficiently navigate from one point to another and what to do if obstacles are encountered along the way. Many map based path planning algorithms attempt to solve this problem, all with varying levels of optimality and complexity. This work shows a review of selected algorithms, and two of these are selected for simulation and testing using a quadrotor unmanned aerial vehicle (UAV) in a dynamic indoor environment which requires replanning capabilities. The Dynamic $A^{*}$ algorithm, or simply $D^{*}$, and the Probabilistic Roadmap method (PRM) are used in a scenario designed to test their respective functionality and usefulness with the goal of determining the better algorithm for flight testing given a partially known or changing environment.

The development of the quadrotor platform hardware is discussed as well as the associated software and capabilities. Both algorithms are redesigned to fit this specific application and display their respective planned and replanned paths in an intuitive and comparable manner. Simulation is performed and an obstacle is added to the map during the quadrotor motion, requiring a replanned path. Results are compared for both computed path length and computational intensity. Flight testing is performed in an indoor environment, and during the flight an obstacle is inserted into the flight path, requiring detection and replanning. Results are compared for computed path length and intuitively analyzed to compare optimality and complexity. 


\section{Acknowledgements}

I would like to thank the following people for their outstanding contributions to my research. I truly could not have done this without you.

Dr. Yu Gu, thank you for believing in me and inviting me to work on your team. Working with you has been an honor and a learning experience every step of the way. Your guidance over the last two years has made me a smarter, harder worker. I have learned much from your quiet openness and intelligence.

Dr. Jason Gross, Dr. John Christian, and Dr. Powsiri Klinkhachorn, thank you for mentoring and guiding me in my pursuit of knowledge. You have all impacted my life and made me want to learn more. Thank you all for being on my defense committee.

A big thank you to everyone associated with the Interactive Robotics Laboratory team: Tanmay Mandal, Kyle Lassak, Yaohui Ding, Jared Strader, Alex Hypes, Caleb Rice, Trenton Larrabee, Scott Harper, Alex Gray, Srikanth Gururajan. You all helped selflessly and encouraged generously. Thank you for your friendship and support.

I also want to thank John Hailer for graciously helping when I was in need. Thank you for the number of hours you selflessly spent to aid me.

Thank you Mom and Dad for always loving me, praying for me and encouraging me in my education from the time I was born. I hope I honor you for putting me on the path to where I am now.

Most importantly, to my loving wife Mikaela, thank you for allowing me to pursue this dream, for being my biggest supporter through it all, and even helping me when I needed extra hands. You inspire me to succeed, and I hope that I will always return the support and love you have shown me.

This research was supported in part by NASA Independent Validation \& Verification Facility and NASA WV Space Grant Consortium. 


\section{Table of Contents}

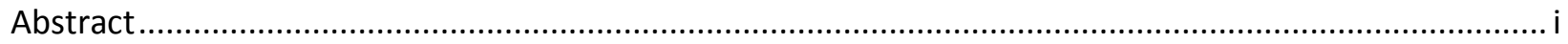

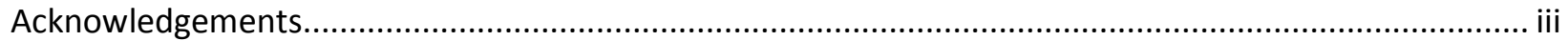

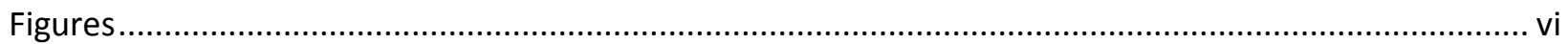

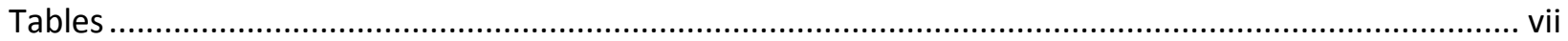

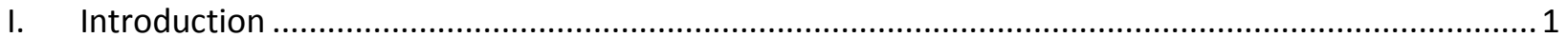

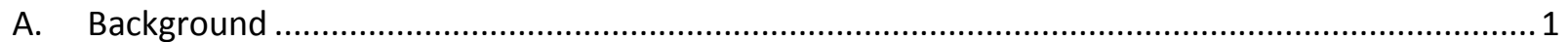

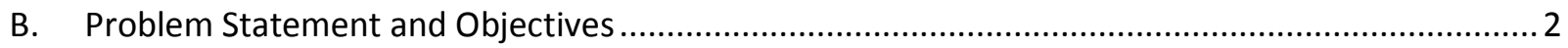

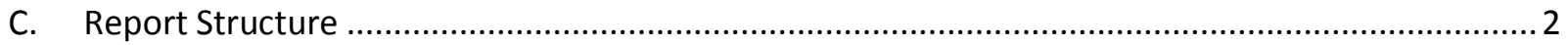

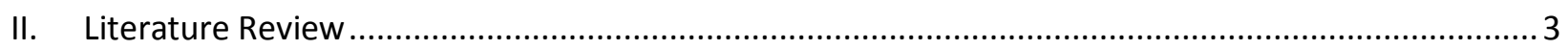

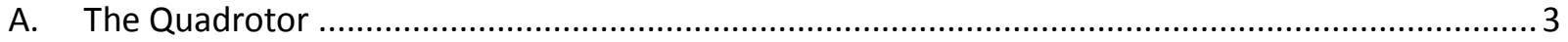

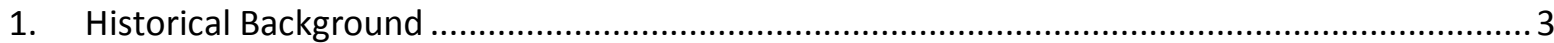

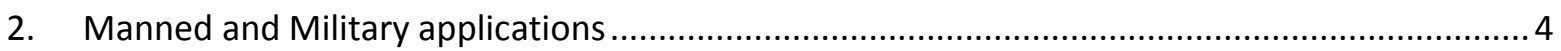

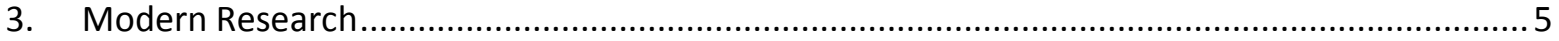

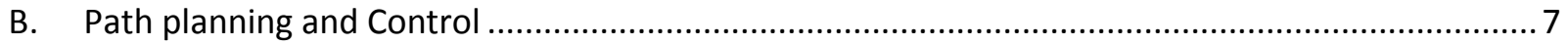

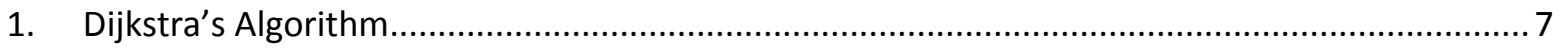

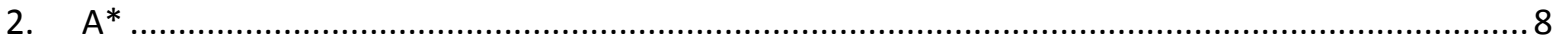

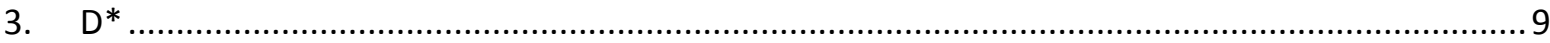

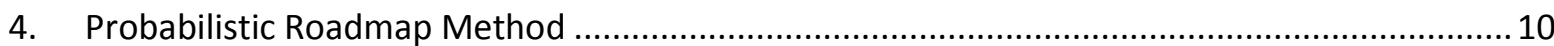

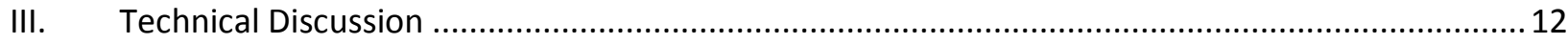

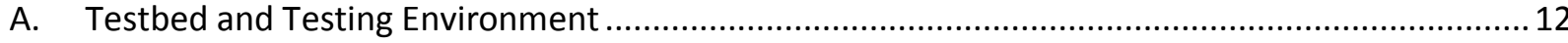

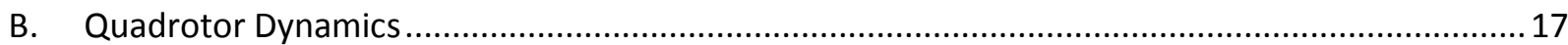

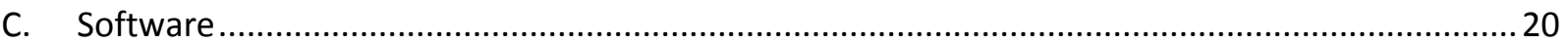

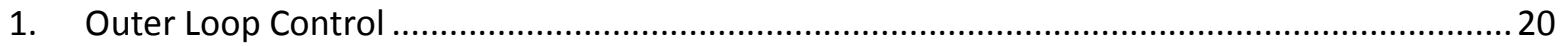

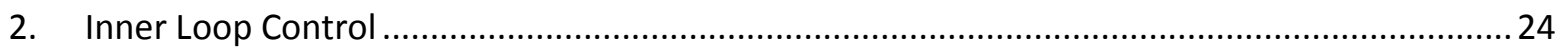

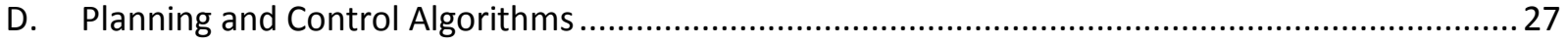

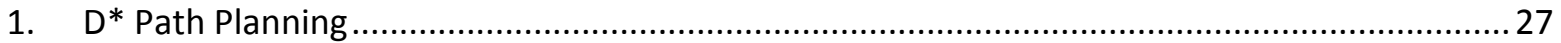

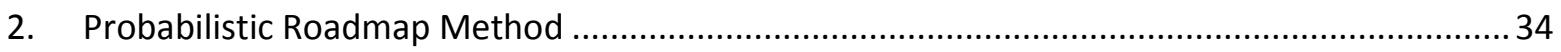

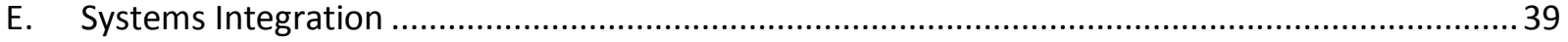

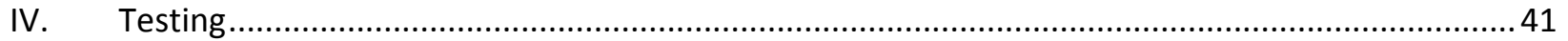




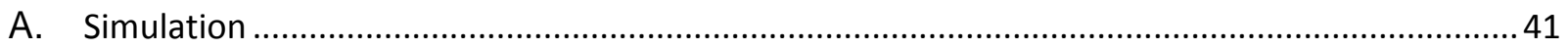

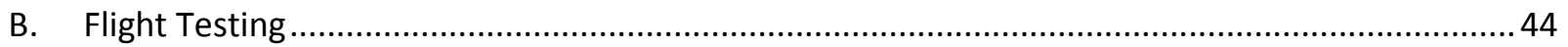

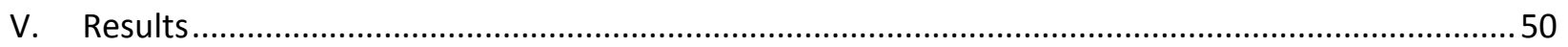

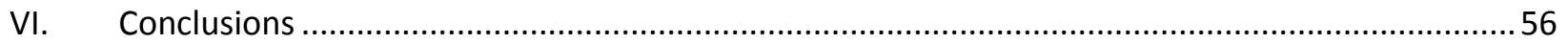

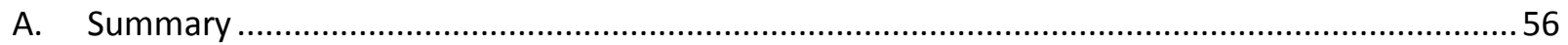

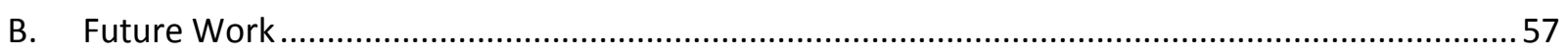

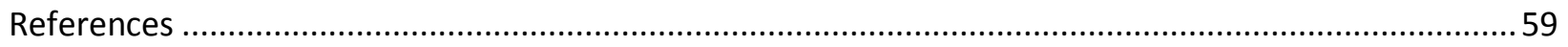




\section{Figures}

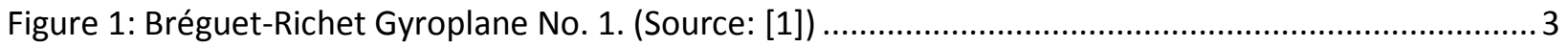

Figure 2: Black Knight Transformer. (Source [5]) ......................................................................... 5

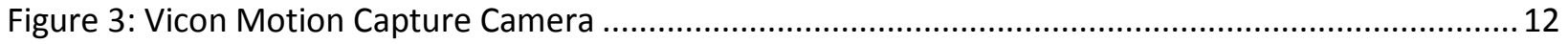

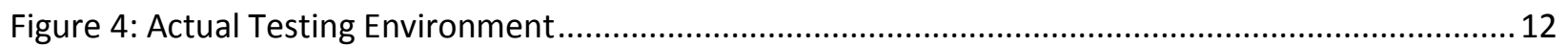

Figure 5: Tracker Visualization of the Testing Environment ................................................................. 13

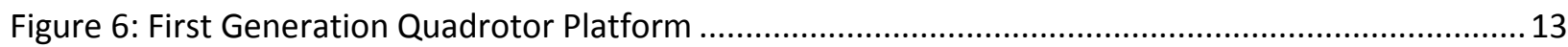

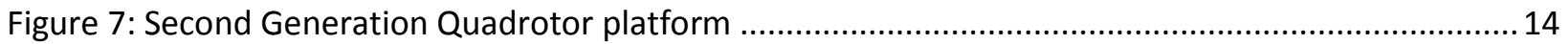

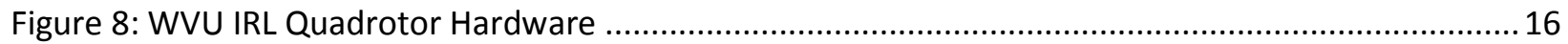

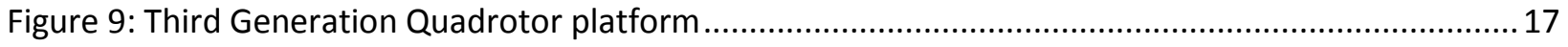

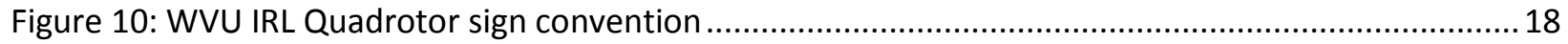

Figure 11: 3D Waypoint Visualization showing waypoints in red and the flight path in blue ...................21

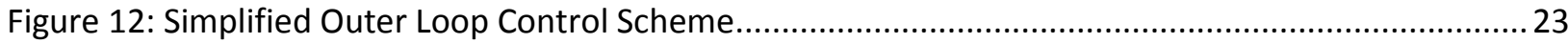

Figure 13: Corke's D* Simulator Costmap and Planned Path (obstacles shown in red)............................28

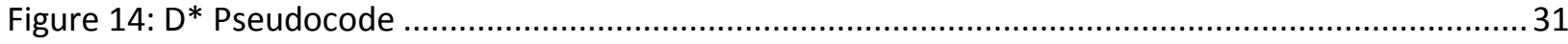

Figure 15: Redesigned $D^{*}$ Algorithm (waypoints shown in blue and buffer zones in white).................... 33

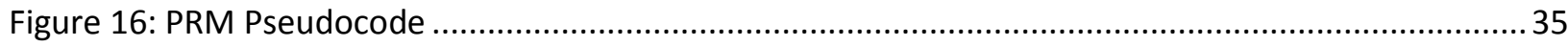

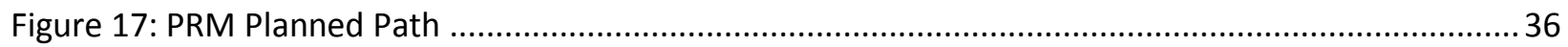

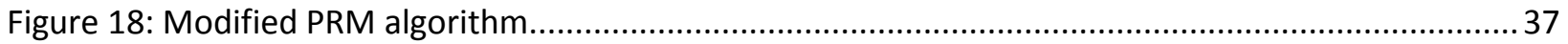

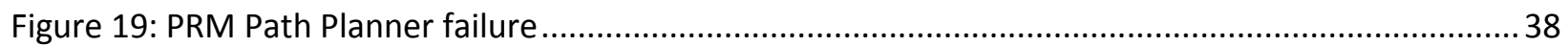

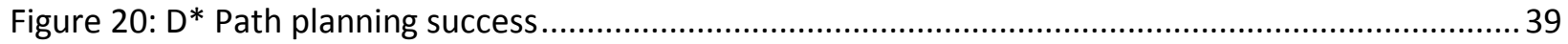

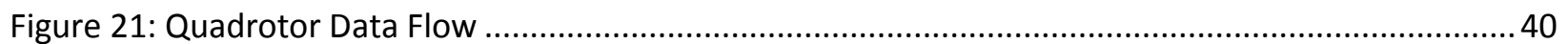

Figure 22: D* Simulation - Planned Path and Unmapped Obstacle .................................................... 41

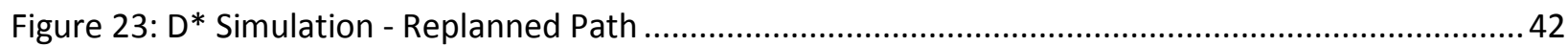

Figure 24: PRM Simulation - Planned Path with Unmapped Obstacle ….................................................4 43

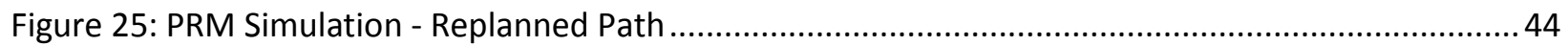

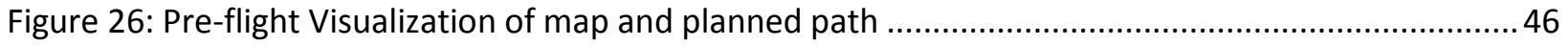

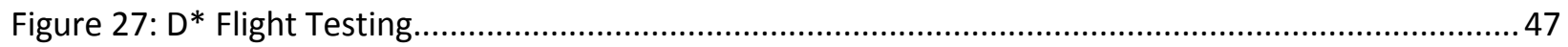

Figure 28: Pre-flight Visualization of map and planned path .............................................................. 48

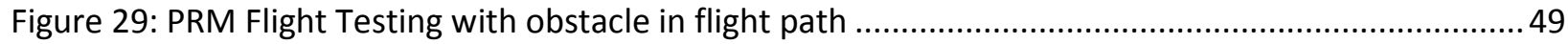

Figure 30: D* Flight Path (in blue) and Replanned Waypoints (in Green) .............................................. 54

Figure 31: PRM Flight Path (in blue) and Replanned Waypoints (in Green)............................................5 55 


\section{Tables}

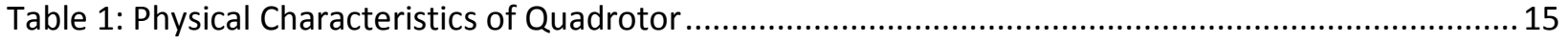

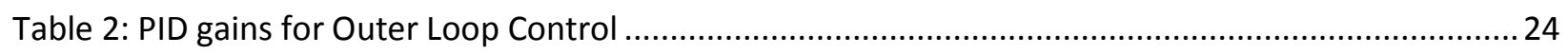

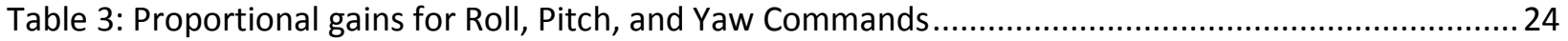

Table 4: Comparison of Path Lengths from Simulation ........................................................................ 50

Table 5: Comparison of time required from Simulation ........................................................................... 51

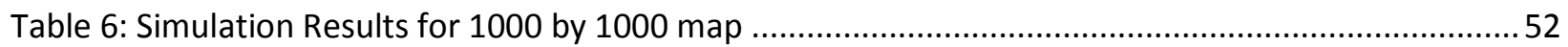

Table 7: PRM time comparison for varied number of nodes in 1000 by 1000 map................................53

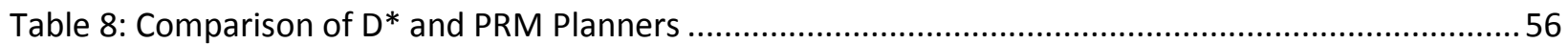




\section{Introduction}

\section{A. Background}

The quadrotor -or quadcopter- aircraft design is a novel vertical takeoff aircraft that is rapidly gaining a foothold in the aircraft research and hobbyist communities. This class of aircraft has numerous advantages that make it an ideal platform for airborne research. A few of these benefits include no control linkages for reduced complexity, inexpensive and readily available parts, battery power for cost efficient energy consumption, as well as vertical takeoff, small size and extreme maneuverability. This has become one of the most widespread flight vehicles used in academia.

Many quadrotors are outfitted with expensive equipment for sensing, navigation, or surveillance. Many are made completely autonomous, extending their applications so that they are not limited by control transmission distance from a human operator. The modification of these aircraft for autonomous flight yields the problems of control and navigation. The desire to traverse from one point to another without damaging the vehicle or its payload necessitates map-based path planning to allow avoidance of obstacles. This is further complicated in real-life scenarios when a map is not completely accurate or the mapped area changes. To meet these needs, a number of replanning algorithms have been created. There are often several variations on each algorithm, and many are case dependent. Real world applicability is severely limited if a quadrotor cannot reliably navigate through such a setting.

Though their uses are varied, it is only when these vehicles are capable of completely independent operation that they live up to their full potential. Therefore, the motivation of this work is to ensure that a quadrotor aircraft can autonomously navigate an inaccurately mapped or dynamic environment in a safe and reliable manner without the intervention of a human operator. 


\section{B. Problem Statement and Objectives}

The goal of the research presented in this report is to design, build, and operate a Vertical Takeoff and Landing (VTOL) quadrotor Unmanned Aerial Vehicle (UAV) that is able to navigate through an indoor environment simulating outdoor conditions, such as a forest, while avoiding unmapped obstacles encountered along the path in real time using a map-based path planning algorithm. The objectives are as follows:

- Two map based path planning algorithms, $\mathrm{D}^{*}$ and Probabilistic Roadmap method, will be integrated and tested on the quadrotor test bed.

- The performance of these algorithms will be analyzed and compared in order to determine the most appropriate method for object avoidance in a cluttered environment.

\section{Report Structure}

The second chapter is a literature review of research relevant to this topic. The third chapter is a technical discussion of the development and structure of the quadrotor aircraft as well as the algorithms to be implemented. The fourth chapter describes the simulation and flight testing procedures used in analysis of the algorithms on the quadrotor test bed. The fifth chapter summarizes the results, and the sixth chapter summarizes and concludes the paper with future work. 


\section{Literature Review}

\section{A. The Quadrotor}

\section{Historical Background}

Despite the recent trend of utilizing the quadrotor aircraft in a variety of applications, this platform is not a new idea. Some of the first aircraft were based on quadrotor designs. The very first quadrotor design ever implemented was in 1907 when French aviation pioneer Louis Bréguet and his brother Jacques, inspired by scientist Charles Richet, built their Bréguet-Richet Gyroplane No. 1 (see Figure 1). This vehicle was a set of four steel-tube girders arranged in a cross with propellers at the four ends of the cross. Each propeller was $8.1 \mathrm{~m}$ in diameter and had four fabric covered biplane surfaces for a total of 32 surfaces to provide thrust. It used an 8 cylinder engine, and weighed approximately $1,200 \mathrm{lbs}$ or more at takeoff. The primary problem with it was that stability was quite poor and as a result was tethered, and the pilot only had control of the throttle of the engine [1].

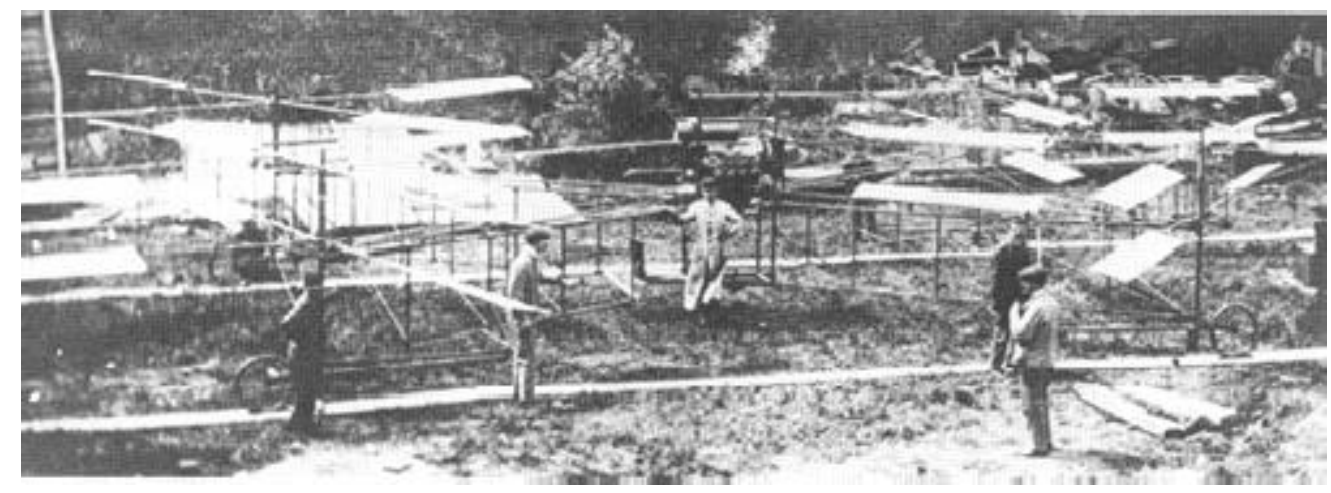

Figure 1: Bréguet-Richet Gyroplane No. 1. (Source: [1])

Over a decade later in 1922, another vertical takeoff aircraft began flying. Built by another Frenchman, Etienne Oehmichen, the Oehmichen No. 2 followed a similar design with four rotors at the ends of a cross. However, for lateral stability Oehmichen used five small horizontal propellers, two more for forward thrust, and a final propeller for steering. The Oehmichen was able to remain airborne for several minutes and established several records at 
the time including the first closed circuit flight of $1 \mathrm{~km}$ by a rotary wing vehicle. Ultimately however, complexity and impracticalities plagued this pioneering machine [2].

There were several other attempts, but as conventional aircraft became more popular little was done with the quadrotor design over the next couple of decades. However, in the mid 1950's a new design was built and tested. The Convertawings Model A Quadrotor, built by Marc Kaplan, was the first true quadrotor. Utilizing only four rotors, the Model A weighed 1 ton and utilized two $90 \mathrm{hp}$ engines. Instead of using another propeller for forward thrust, it varied the thrust of the four main rotors instead, creating moments around the center of gravity that were used for control. While this design was largely successful, fixed wing aircraft were preferred in nearly every category such as range and speed, in addition to the fact that the pilot's workload was much lighter [3].

\section{Manned and Military applications}

There have been a few attempts in recent years to make full-scale manned multirotor aircraft, but they are not very common due to numerous technical difficulties. Electric propulsion was usually not powerful enough, and typical combustion engines cannot change RPM fast enough to be practical for full size quadrotor applications [4]. Engine failure on a manned flight might also be catastrophic. However, there are several companies that are pushing these boundaries with variations on the quadrotor design. The first is Advanced Tactics and their Black Knight Transformer aircraft which employs eight engines instead of four (see Figure 2). It can travel on the ground or through the air, and is designed to be a cargo or medivac transport vehicle more flexible than its counterparts [5]. 


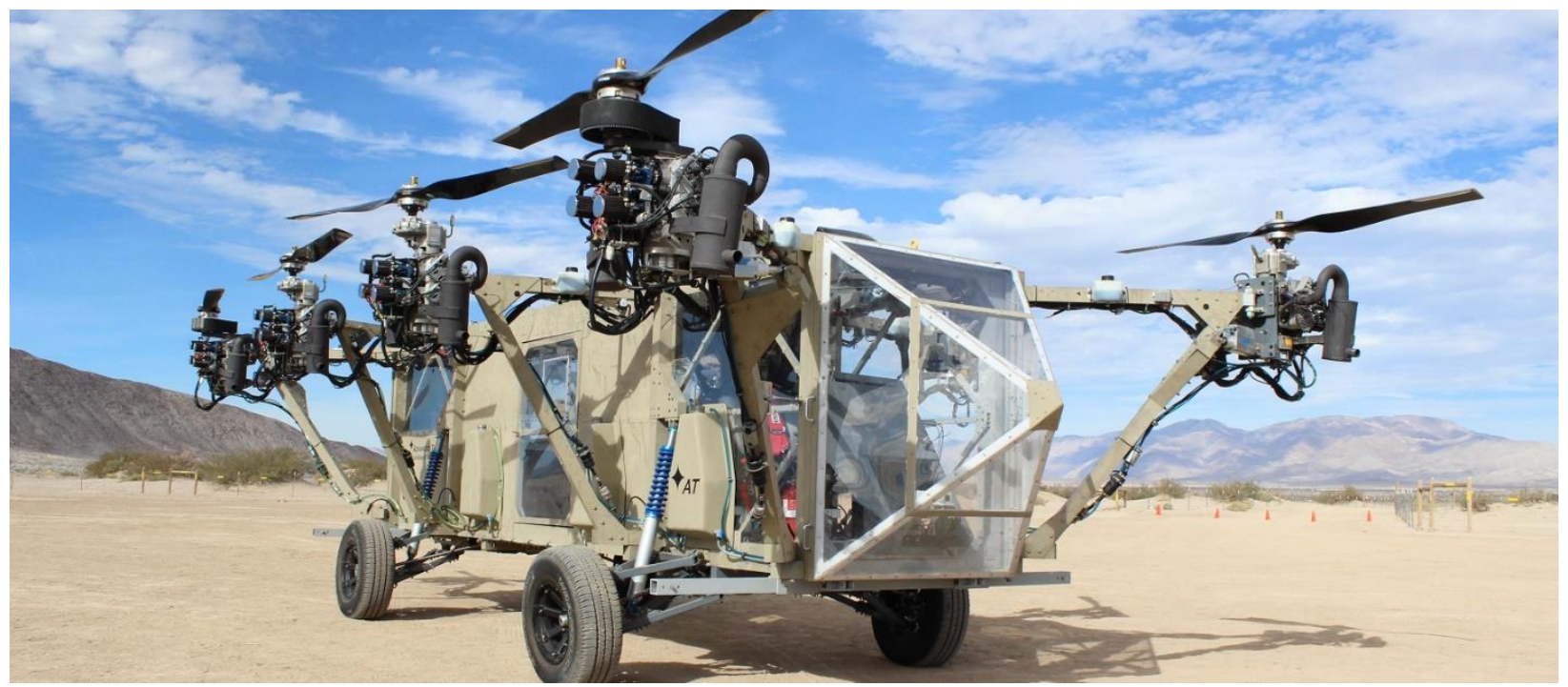

Figure 2: Black Knight Transformer. (Source [5])

Other manned vertical takeoff designs focus more on personal transportation. The German company E-volo has created the VC200 Volocopter, which is a small, two person private helicopter with 18 electric motors providing the thrust and controlling it similarly to a quadrotor [6]. The Malloy Aeronautics Hoverbike is a smaller, and can be folded down to one third of its length for easy transportation. This platform is now also being further developed for the U.S. Department of Defense for use in personal transportation or surveillance [7] [8]. Finally, Joby Aviation has created the S2, which uses a conventional aircraft body but is a VTOL two person aircraft with 12 electric motors mounted on the leading edges of the wings and tail [9].

3. In addition to manned flights, many have seen the extensive potential of these adaptable aircraft for military applications. One such application proposes to use a quadrotor to investigate culverts in warzones in order to ensure that there are no improvised explosive devices hiding beneath roadways [10]. An interesting concept with potential military or naval exploration applications has a quadrotor landing on a marine vehicle, even in light winds and currents [11].Modern Research

As the quadrotor platform has increased in popularity in recent years, there has been more research into various designs and applications for quadrotors. The first true modern research quadcopter was the HoverBot [13]. This aircraft was made in 1992, and was the first to 
overcome the inherent instability of small-sized helicopters using onboard computing and electronic power. At that time, electronics were just becoming small and powerful enough to fit such an application, and batteries were beginning to have larger power-to-weight ratios. However a typical Remote Controlled $(\mathrm{RC})$ helicopter could not produce the required power to support the extra weight from the electronics. Thus the quadrotor design was chosen, and essentially implemented as four helicopters linked by the tails. Their design allowed for tethered indoor flight, another first for the quadrotor. Despite the modest results, this was a large step forward in the evolution of modern quadcopters.

Since that time, many other fascinating projects utilizing the flexibility of the quadrotor have embodied themselves, often related to surveillance. Since flight times are still relatively short however, a major area of study is extending the duration of surveillance missions to increase effectiveness. A common way to achieve this is to "perch-and-stare," where the quadrotor lands on a suitable surface to save battery power while a camera continues surveillance. One group has built a quadrotor that can perch on a large variety of surfaces while using almost no power by utilizing a bio-mimetic mechanism [19]. In addition to vertical landing, actuators may also be used to expand a quadrotor's capabilities to interact with the environment in other ways, to pick up other objects or even perch upside down [21]. While this is potentially very useful, a quadrotor equipped with such an actuator still needs to be controlled precisely to be able to utilize this. To achieve this, the University of Pennsylvania's GRASP lab has developed an algorithm which allows a quadrotor to perch on an arbitrarily oriented line [20]. Another research aircraft that could be useful for such surveillance applications is the SquidCop. This quadcopter aims to increase the flight time it can maintain at its mission site, and achieves this by deploying from a fixed wing carrier aircraft at low speeds. This allows the quadrotor to use little or no power reaching the surveillance point [12].

Another very popular research topic with quadrotor aircraft is cooperative swarms which can achieve much more than a single aircraft. With this trend comes the need for simple, low 
cost platforms to facilitate large numbers of quadrotors flying concurrently. Electronics are constantly becoming cheaper and more readily available, building swarms of quadrotors and equipping them with electronics and sensors possible [27]. Many algorithms have been made to control swarms of autonomous vehicles using coordinated formations, path planning and object avoidance procedures [28] [29] [30] [31] [32]. These capabilities can be used for surveillance patrols or other applications where numerous vehicles are in close operational proximity to each other. Some have even extended the use of these swarms to perform tasks in areas dangerous to humans. Penn's GRASP lab and the Technological Institute of Aeronautics in Brazil both have attached grippers to their quadrotors to assemble a variety of geometric truss structures using special assembly members in a construction scenario [33] [34]. Another hazardous application for swarms includes mass deployment in disaster zones for expanding telecommunication and WiFi signals [35].

There are several other more creative cooperative applications for quadrotors that have been investigated and implemented at ETH Zurich in Switzerland. They include carrying a large flexible payload similar to a hula hoop [36], throwing and catching a ball using a net [37], and even execute aerial dance choreography in time with music [38]. Control can also be achieved using a Microsoft Kinect sensor to directly interact with the quadrotor simply using motions [39]. Additionally, they have experimented with a modular, single propeller robot that can autonomously drive around the room, dock with other robots, and fly in a coordinated fashion while connected. [40]

\section{B. Path planning and Control}

\section{Dijkstra's Algorithm}

One of the foundational modern map-based planning algorithms is Dijkstra's Algorithm, published in 1959. Named after its Dutch creator, this algorithm solves the problem of the shortest total length between a start and goal node given the lengths of all branches that 
connect the nodes. It can also be used to find the shortest path from the start node to all other nodes. It takes into account only the neighboring nodes and then moves from the current point to the next "best" point. It will not return to any node it has already visited and will continue until the destination is reached [41]. It is very efficient since each iteration of the algorithm only relies on previously determined information. Due to its efficiency it is one of the most widely used methods for finding the shortest path in a digraph [42].

This algorithm has obvious uses in robotics and path planning for autonomous vehicles [43] [44], but it has also been used in a variety of other interesting applications through the years. One group discusses using a modified version to route emergency vehicles in natural disaster areas [45], and another uses it for airline network planning [46]. Dijkstra's algorithm has even been utilized to evaluate a 3D skeletonization of the brain's vascular network [47].

Despite its advantages, Dijkstra's Algorithm is very hard to parallelize, or run concurrently on multiple devices for increased speed. Some work has been done to parallelize it using transactional memory and helper threading, which shows some moderate improvements to the basic serial algorithm [48] [49]. Others have made improvements to the algorithm by considering node weights in addition to the edge weights [50], or improving its storage structure and search area [51]. Some go as far to argue that Dijkstra's Algorithm should not be used at all any more, replacing it with the Uniform-Cost Search method which is very similar but superior to Dijkstra's Algorithm [52].

2. $\mathbf{A}^{*}$

In 1967 another important algorithm was made called A* (pronounced "A-Star") which improved upon Dijkstra's Algorithm by using heuristics to weight the search towards the goal node. This algorithm is admissible, meaning it is guaranteed to find an optimal path to the goal node, and will process the fewest nodes necessary to find the optimal path [53]. There are many similar applications of $A^{*}$, thus it has been compared to Dijkstra's Algorithm frequently [54]. 
Though it generally achieves good results, the complexity can be a drawback for larger maps. As a result, there have been several improvement or variations off of the $A^{*}$ algorithm as well [55] [56]. One of these is called Ae, which is not admissible but, rather than seeking an optimal solution, will look for one that is close to optimum. The idea is that increased computational efficiency may be achieved at the cost of a bounded loss in optimality [57]. Another adapts $A^{*}$ for use in areas where a "large" map is necessary, reducing the necessary processing resources [58].

3. $\mathbf{D}^{*}$

Another major map based planning algorithm in history is based off of $A^{*}$. Created by Anthony Stentz in 1994, Dynamic $A^{*}$, or simply $D^{*}$, extends $A^{*}$ planning to include replanning in unknown or changing environments while retaining optimality and efficiency. It can handle any amount of map information from none at all to a complete and accurate map [59]. Stentz also made several variations on his algorithm. The first of these is the Focussed $D^{*}$ algorithm which reduces the time needed for path planning and replanning. After the initial plan, the algorithm then changes the path as needed during the execution of the plan, still following an optimal path at every step [60]. Another $\mathrm{D}^{*}$ algorithm modification was made by Koenig and Likhachev, who called their new algorithm $D^{*}$ Lite. $D^{*}$ Lite is essentially the same algorithm as Focussed $D^{*}$ but is easier to understand and more efficient [61]. After these changes, another modification was made by Stentz to the algorithm, this time called Delayed $D^{*}$. This algorithm is simply more efficient in how it modifies the map during the mission making it superior to even $D^{*}$ Lite [62]. Stentz et al also worked to combine $D^{* \prime}$ incremental replanning with an anytime algorithm, or one that will calculate the best solution within the available time since generating an optimal solution may not be feasible. The result is known as Anytime Dynamic $A^{*}$, which takes the best qualities form both types of planners to give solutions to complex and changing environments [63]. 
The $D^{*}$ family of algorithms is used across a spectrum of applications, ranging from an enhanced version of the basic algorithm for basic ground robot navigation in indoor environments [64] to simulating flights of unmanned combat aircraft [65]. They also can be integrated with other algorithms to provide practical improvements such as reactive collision avoidance in addition to path planning [66] or to smooth the path generated by $\mathrm{D}^{*}$ [67].

For grid based algorithms such as $A^{*}$ and $D^{*}$, the generated path is optimal. However optimality is only guaranteed to be as good as the grid resolution of the map, and as the grid grows run-time increases exponentially [68]. This problem may potentially be remedied by using different methods, particularly roadmap methods.

\section{Probabilistic Roadmap Method}

One of these algorithms is named the Probabilistic Roadmap method, or PRM, which is part of the sampling-based roadmap family of algorithms. This method is built for motion planning for holonomic robots - robots that may move in any direction - in static spaces, and is easy to understand and implement. It has two phases: the learning phase in which the map is built, and the query phase in which the path through the map is planned. In the learning phase, points are chosen at random and set as nodes. Edges are then added between these nodes to denote they are passable, which is termed "local planning" [69]. In the query phase a path is chosen between the nodes. Occasionally this is achieved using Dijkstra's algorithm [70], [71], [72], [73], but can be through other methods as well.

One of the major drawbacks to PRM's is the fact that they often will not perform well in narrow passages, sometimes not even arriving at a navigation solution if node selection is inadequate. Modifications have been made to the learning phase of the planner to take into account these locations and ensure proper sampling in these areas [72], [73], [74], [75]. One method even allows several learning techniques to be stored, and chooses the correct one for a given application [76]. 
The PRM method has been used for trajectory generation in three dimensions for aircraft [77], multiple robot configurations [78], as well as other general mobile platforms [71]. One technique alters the generated nodes in order to create more practical, less random paths [79]. Another attempts to parallelize the PRM method [80]. Work has even been done to allow PRM's to take into account roadmap inaccuracies by adding a probability of collision to the edges and nodes [70].

The PRM algorithm is known to be suboptimal, due to the random nature of the node selection. However, there are several alternative methods based on the PRM algorithm that provide optimality. Some of these also retain very good computational efficiency [68] which may make these methods a viable alternative to grid based planning algorithms. 


\section{Technical Discussion}

\section{A. Testbed and Testing Environment}

The West Virginia University Interactive Robotics Laboratory outfitted an indoor flight testing facility specifically for quadrotor UAVs. The testing area is roughly a $4 \times 4 \times 2 m$ room with antireflective paint and a foam ground mat. Safety equipment includes sprinkler guards and a safety

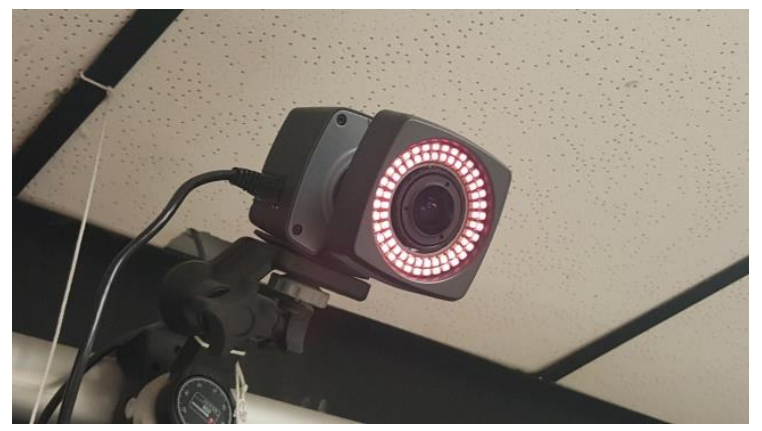

Figure 3: Vicon Motion Capture Camera net to provide protection to the room, the pilot, and bystanders during indoor quadrotor flight testing. A Vicon infrared motion capture camera system (see Figure 3) was installed along with Vicon's Tracker software. This system allows real-time millimeter accuracy three-dimensional position tracking of multiple objects at a high frequency through tracking infrared reflective spheres attached to the subject(s) of interest. A unique orientation of the reflectors is required to define an object to the Tracker software so that it may differentiate between the objects in the workspace (see Figure 4 and Figure 5). In addition, Quarc Real-Time control software is utilized with MATLAB and Simulink in conjunction with Tracker to allow real-time collection and analysis of Vicon position data on a ground station computer.

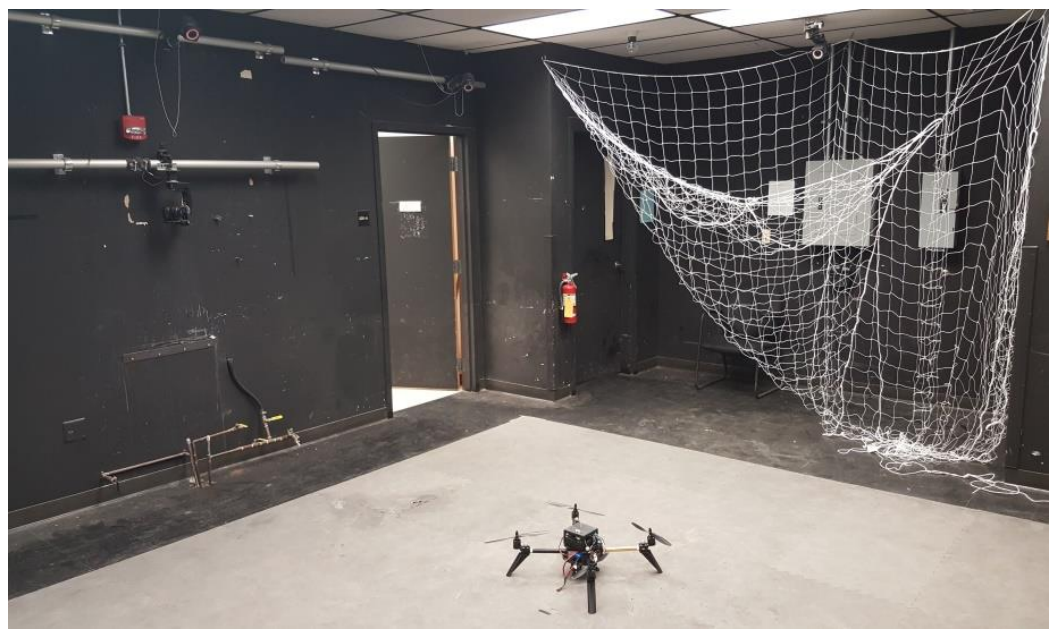

Figure 4: Actual Testing Environment 


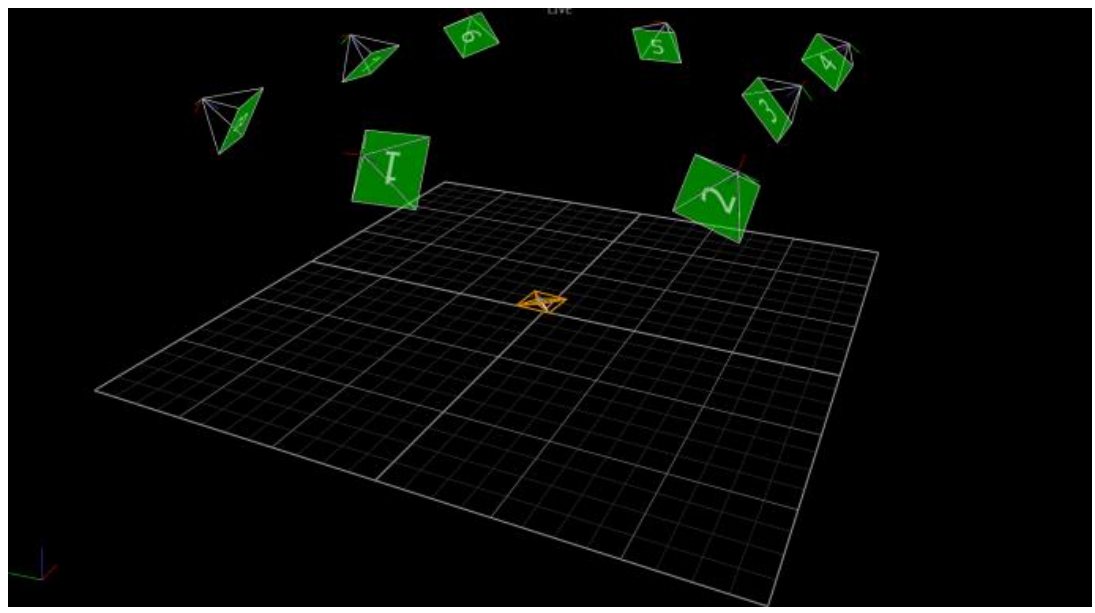

Figure 5: Tracker Visualization of the Testing Environment

Throughout the lifetime of this project, three different quadrotor frames have served as the three distinct generations of the testing platform. Initially, a DJI Flamewheel quadrotor was set up to fly using simple Radio Controls $(\mathrm{RC})$ and off-the-shelf motors, propellers and a controller. The controller compiled Inertial Measurement Unit (IMU), GPS, and RC data, and then output a specific voltage to the motors. The motors used for this frame were relatively weak and came with eight inch diameter propellers. This setup was then duplicated using another offthe-shelf kit. Several other quadrotor frames were purchased and built to serve as backups. The first generation testing quadrotor was then developed by incorporating a custom avionics board onto one of these frames (see Figure 6). The avionics were crudely mounted using a simple cardboard box attached to the top of the quadrotor frame. Ten inch propellers were used with this configuration in order to provide extra lift at the cost of reduced flight time to accommodate the heaver electronics. In addition, reflective markers were attached to the quadrotor so that it could be used with the Vicon system, and an XBee

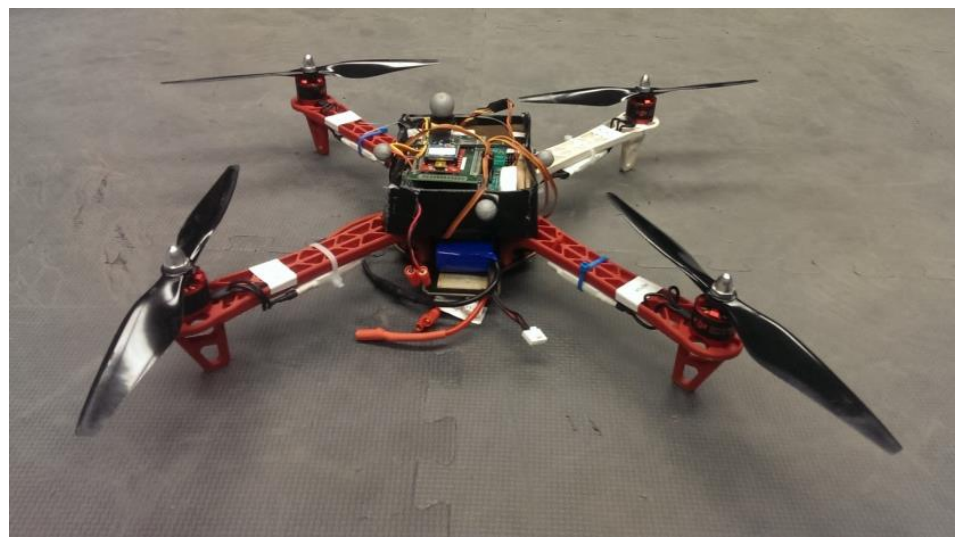

Figure 6: First Generation Quadrotor Platform 
wireless RF module was connected to the electronics to create a serial interface with another Xbee connected to the ground station computer for closed loop control feedback.

As the project progressed, it became apparent that another quadrotor platform was needed to increase the total sensor payload. As a result, the second generation quadrotor was built on a larger Century NEO 600 frame with larger motors and twelve inch propellers (see Figure 7).

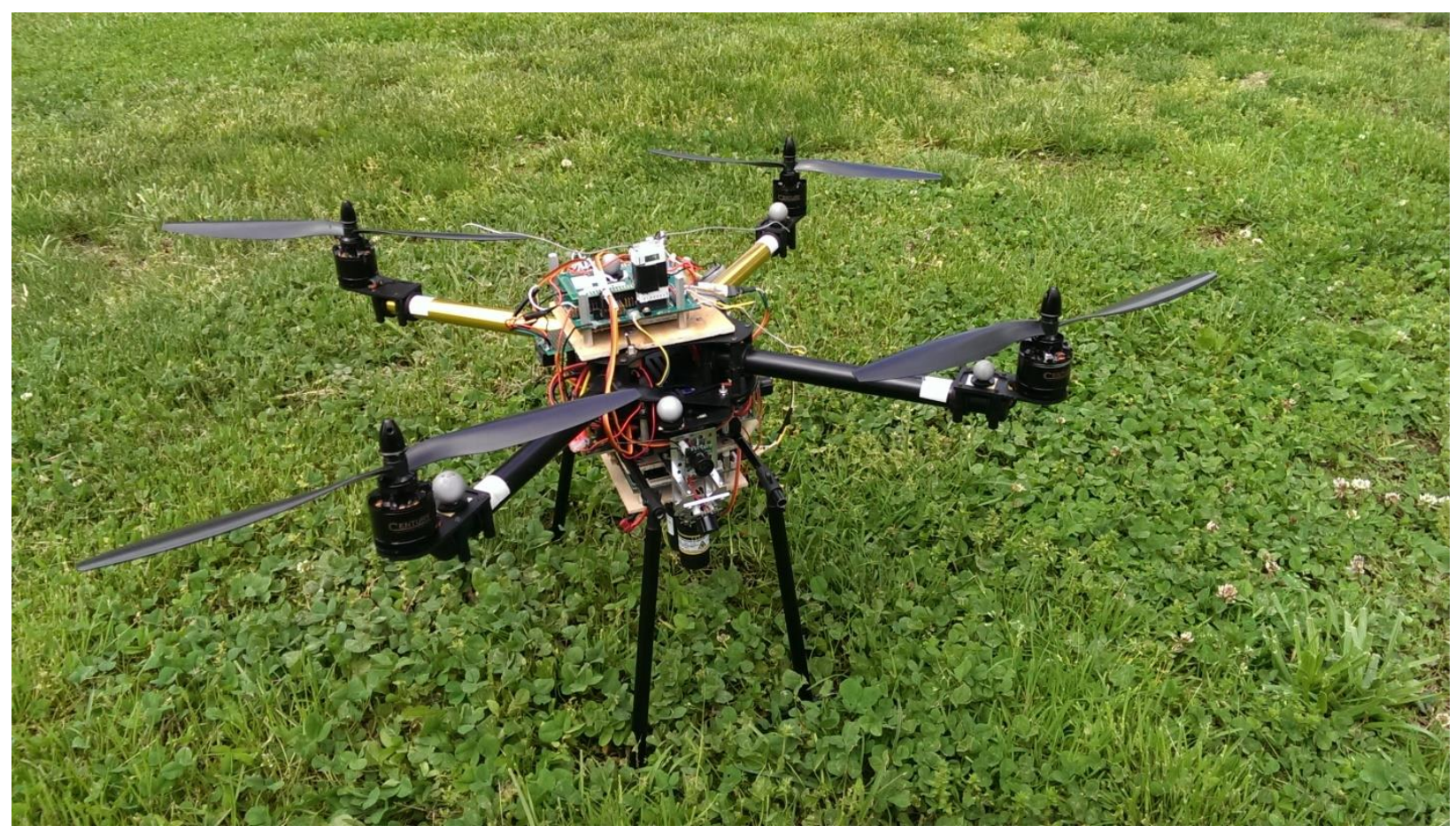

Figure 7: Second Generation Quadrotor platform

This newer quadrotor used an improved and updated second-generation avionics board and was compatible with a Novatel GPS receiver and an Advantech PC104 CPU module. The PC104 module can run Linux from a compact flash card and was implemented in order to allow the outer loop Simulink control scheme to be run directly on the quadrotor by using an older version of MATLAB, MATLAB 7.0.1. This was to allow all computations and control to be performed on the quadrotor platform so that it will not need a ground station to fly.

Initially the PC104 module was mounted on top of the quadrotor with the autopilot board, but after extensive testing and troubleshooting it was determined that this configuration was 
unable to be controlled. This was due to the fact that the center of gravity was too high, therefore making the quadrotor inherently unstable, and the IMU was too far from the center of gravity. Both of these problems were corrected by suspending the PC104 module beneath the quadrotor. This lowered the center of gravity and greatly enhanced the stability of the quadrotor.

Once the PC104 had been relocated, flight testing proved that manual RC stable flight could be achieved. Next, trials were done using the same method as described above without utilizing the PC104 to ensure autonomous flight was possible using the same inner-loop controller and outer-loop control scheme. This approach worked quite well, and so testing proceeded to incorporating the PC104 into the control loop.

Finally, a third generation quadrotor was developed to clean up and finalize much of the hardware into a more concise and robust flight test platform. This hardware is outlined below (see Table 1 and Figure 8):

Table 1: Physical Characteristics of Quadrotor

\begin{tabular}{|c|c|c|c|}
\hline Max Dimensions & Weight & Propeller Size & Avg. Flight Time \\
\hline $0.53 \times 0.51 \times 0.23 \mathrm{~m}$ & $1.81 \mathrm{~kg}$ & $0.30 \mathrm{~m}$ & $300 \mathrm{sec}$ \\
\hline
\end{tabular}




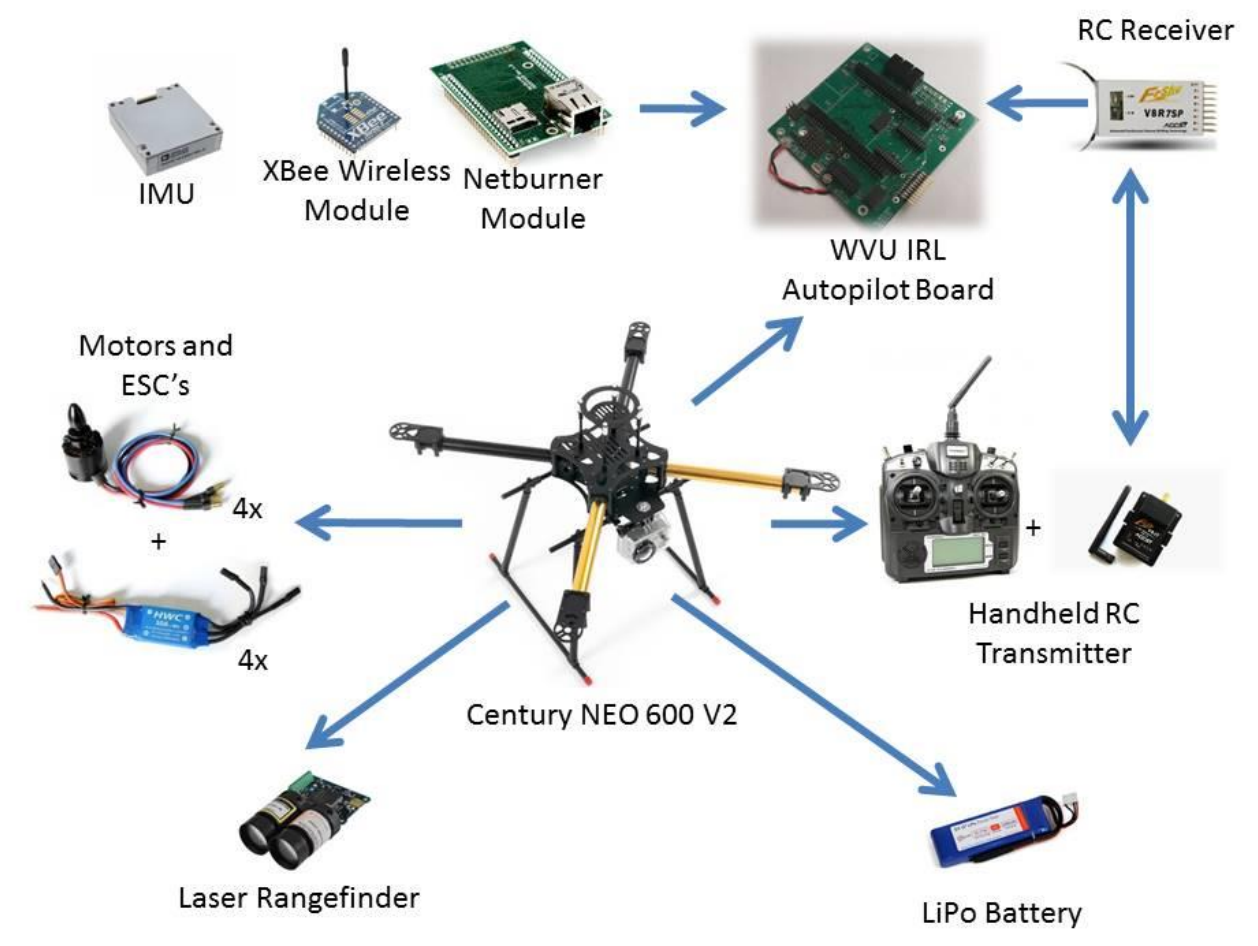

Figure 8: WVU IRL Quadrotor Hardware

The design is much more streamlined, with new third-generation avionics mounted in a custom 3D printed housing and fewer loose wires cluttering the quadrotor. In addition, 3D printed landing gear were designed and built so that, if needed, more could be made in-house. The quadrotor uses the same battery, motors and twelve inch propellers as the previous generation.

In terms of sensor payload, this quadrotor uses a simple laser rangefinder for object detection and avoidance. A LightWare SF02/F laser rangefinder is mounted on the top of the quadrotor facing forward so that there are no obstructions from any other part of the quadrotor. This sensor has a theoretical maximum range of fifty meters with one centimeter resolution. It has been tested and proven to be highly accurate and reliable for object detection (see Figure 9). 


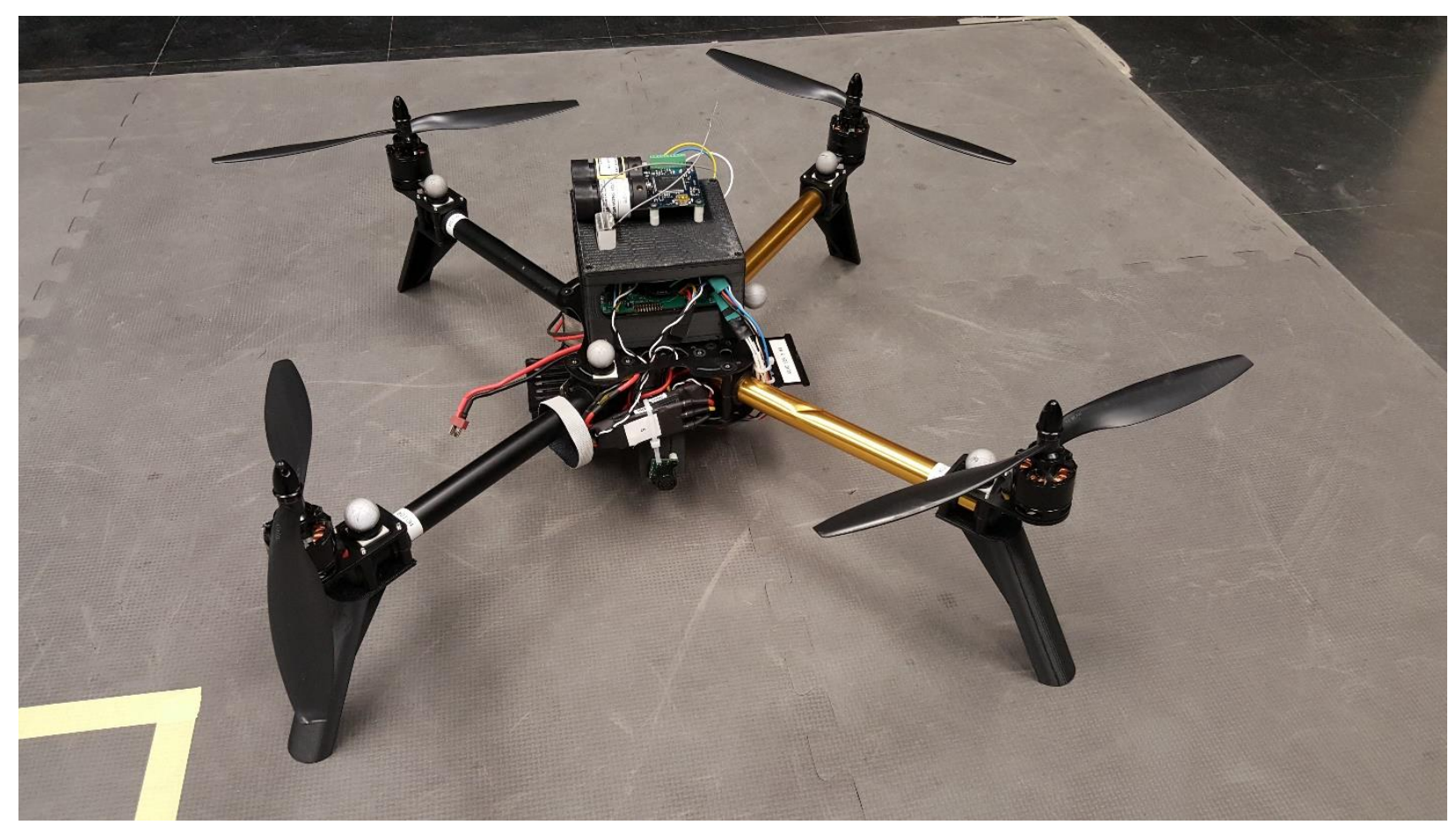

Figure 9: Third Generation Quadrotor platform

\section{B. Quadrotor Dynamics}

The quadrotor aircraft design and control is very intuitive. There are two possible configurations for a quadrotor, named the " $X$ " and "cross" configurations, respectively, both named for their shape. For the "X" configuration, which is used in this research, there are two pairs of counter-rotating propellers mounted on motors at the four ends of the " $X$ ", with opposite blades spinning the same direction (see Figure 10). Translation cannot occur without rolling or pitching actuation, in order to direct a portion of the quadrotor's thrust in the desired direction of motion. To hover, the quadrotor's motors all generate the same amount of thrust. To roll to the right, motors 2 and 3 increase thrust, while motors 1 and 4 decrease thrust in order to keep the same altitude during the maneuver. The same concept applies to the pitch axis. To yaw, motors an opposing pair of propellers' thrust is increased while the other propellers decrease, and the resulting torque differential will rotate the quadrotor in place. 


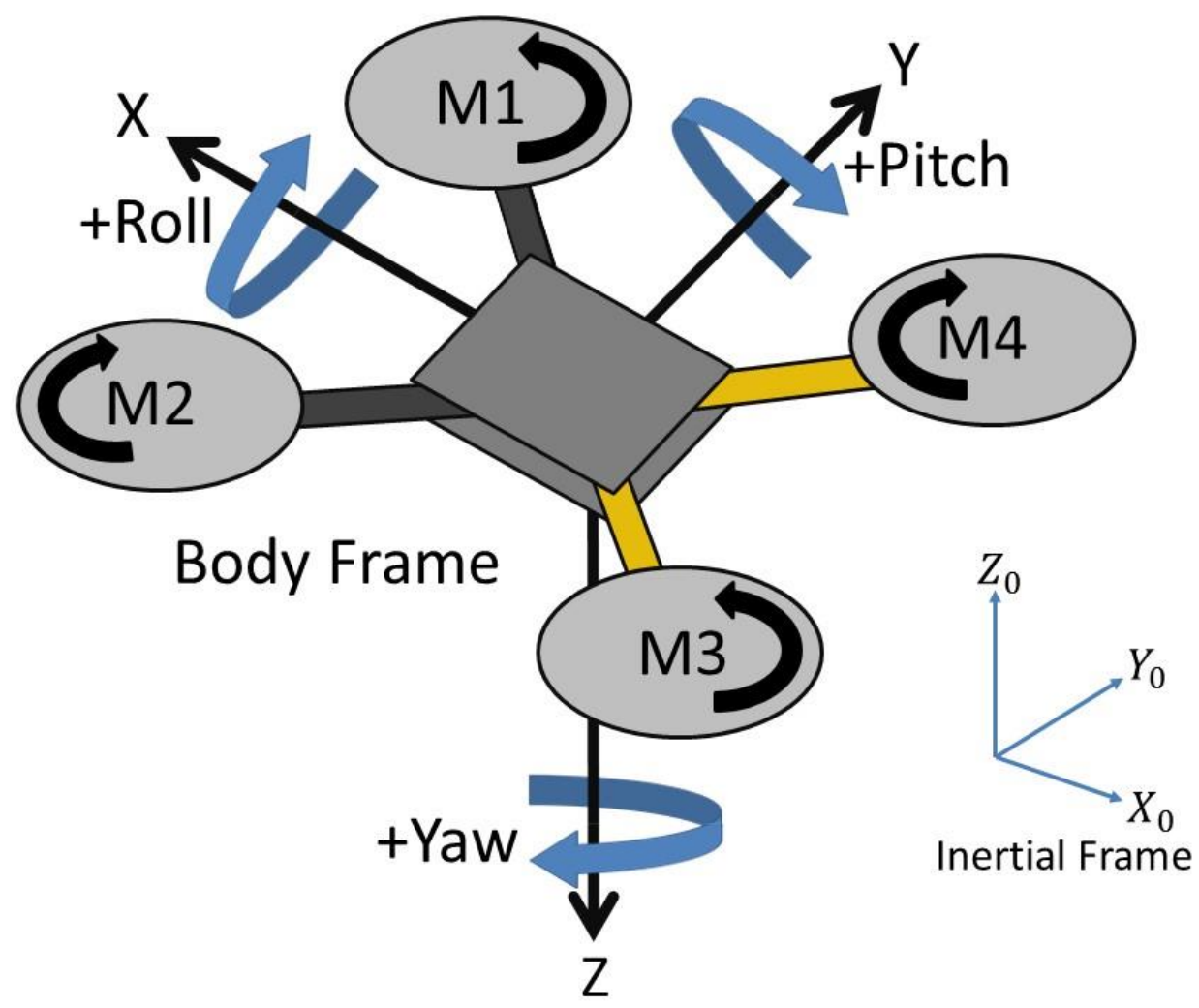

Figure 10: WVU IRL Quadrotor sign convention

Two frames of reference must be taken into account, the Earth Fixed Inertial or Global frame, and the Body or Local frame. To convert from the inertial reference frame to the body frame using Euler angles, the direction cosine matrix $R$ is used in Equation 1 below.

$$
R=\left[\begin{array}{ccc}
c(\psi) c(\theta) & c(\psi) s(\theta) s(\phi)-s(\psi) c(\phi) & c(\psi) s(\theta) c(\phi)+s(\psi) s(\phi) \\
s(\theta) c() & s(\psi) s(\theta) s(\phi)+c(\psi) c(\phi) & c(\psi) s(\theta) s(\phi)-s(\psi) c(\phi) \\
-s(\theta) & s(\psi) c(\theta) & c(\psi) c(\theta)
\end{array}\right]
$$

Where $c(x)=\cos (x)$ and $s(x)=\sin (x), \phi$ is roll angle, $\theta$ is pitch angle, and $\psi$ is yaw angle. Using the Newton-Euler formulation for the rigid body model of the quadrotor, we have the inertial frame $\mathrm{I}=\left[\mathrm{e}_{\mathrm{x}}, \mathrm{e}_{\mathrm{y}}, \mathrm{e}_{z}\right]$ and the body frame $\mathrm{A}=\left[\mathrm{e}_{1}, \mathrm{e}_{2}, \mathrm{e}_{3}\right]$, the model is described as follows in Equations 2-6 below.

$$
p \doteq v
$$




$$
\begin{gathered}
\dot{v}=g e_{z}-\frac{1}{m} T R e_{z} \\
\dot{R}=R S(\Omega) \\
I_{f} \dot{\Omega}=-\Omega \times I_{f} \Omega-G_{a}+\tau_{a} \\
I_{r} \dot{\omega}_{i}=\tau_{i}-Q_{i}, \quad i \in\{1,2,3,4\}
\end{gathered}
$$

Where $p$ is the $x, y, z$ origin positon of the body frame with respect to the inertial frame, $v$ is the velocity of the origin of the body frame, $g$ is acceleration due to gravity, $m$ is mass of the quadrotor. For the vector $\Omega=\left(\Omega_{1}, \Omega_{2}, \Omega_{3}\right)^{\top} S(\Omega)$ is the skew-symmetric matrix defined in Equation 7 below.

$$
S(\Omega)=\left[\begin{array}{ccc}
0 & -\Omega_{3} & \Omega_{2} \\
\Omega_{3} & 0 & -\Omega_{1} \\
-\Omega_{2} & \Omega_{1} & 0
\end{array}\right]
$$

Torque $Q$ is generated due to the drag forces of each of the rotors acting on the air. This is modeled by Equation 8 :

$$
Q_{i}=\kappa \omega_{i}^{2}
$$

Thrust $T$ is the sum of the thrust from all motors, shown with Equation 9.

$$
T=b \sum_{i=1}^{4} \omega_{i}^{2}
$$

Where $\kappa$ and $b$ are coefficients varying with propeller shape, pitch, size, etc. Gyroscopic torque from airframe and rotor rotation is given by:

$$
G_{a}=\sum_{i=1}^{4} I_{r}\left(\Omega \times e_{z}\right)(-1)^{i+1} \omega_{i}
$$

The airframe torque $\tau_{a}$ due to the rotors are from the following equations: 


$$
\begin{gathered}
\tau_{a}^{1}=d b\left(\omega_{2}^{2}-\omega_{4}^{2}\right) \\
\tau_{a}^{2}=d b\left(\omega_{1}^{2}-\omega_{2}^{2}\right) \\
\tau_{a}^{3}=\kappa\left(\omega_{1}^{2}+\omega_{3}^{2}-\omega_{2}^{2}-\omega_{4}^{2}\right)
\end{gathered}
$$

Where $d$ is the length from the origin of the body frame at the center of mass of the quadrotor to the rotor. These can be combined as shown below in Equation 12.

$$
\tau_{a}=\left[\begin{array}{c}
\tau_{a}^{1} \\
\tau_{a}^{2} \\
\tau_{a}^{3}
\end{array}\right]
$$

Lastly, the torque produced by a given rotor is denoted by $\tau_{i}[81]$.

\section{Software}

\section{Outer Loop Control}

An outer loop control scheme was designed in Simulink to be run on the ground station computer using feedback from the Vicon system and Quarc software to control the quadrotor. The basic scheme applied with this system is a simple waypoint navigation controller. It employs a Matlab script to initialize desired position, which generates a user-friendly Graphical User Interface (GUI) that guides the user in choosing waypoints around the test area in threedimensional space. An imaginary sphere of a specific radius is then placed around those points. The final set of waypoints and spheres can be viewed in 3D with a ground trace for enhanced perspective. These waypoints are then output as a matrix of $x y z$ coordinates into Simulink. Simulink will control the quadrotor as described above using a constant yaw angle and the first waypoint's xyz coordinates as the current desired location. When the quadrotor enters any part of the sphere around the waypoint, the control scheme then accepts that the waypoint was reached and updates the desired location to the next waypoint, and so on. When the last waypoint is reached then the next waypoint loops back to be the initial waypoint so that flight is 
continuous until the pilot lands the quadrotor. After flight the quadrotor's path can be plotted in the 3D perspective Matlab plot of the plan (see Figure 11)

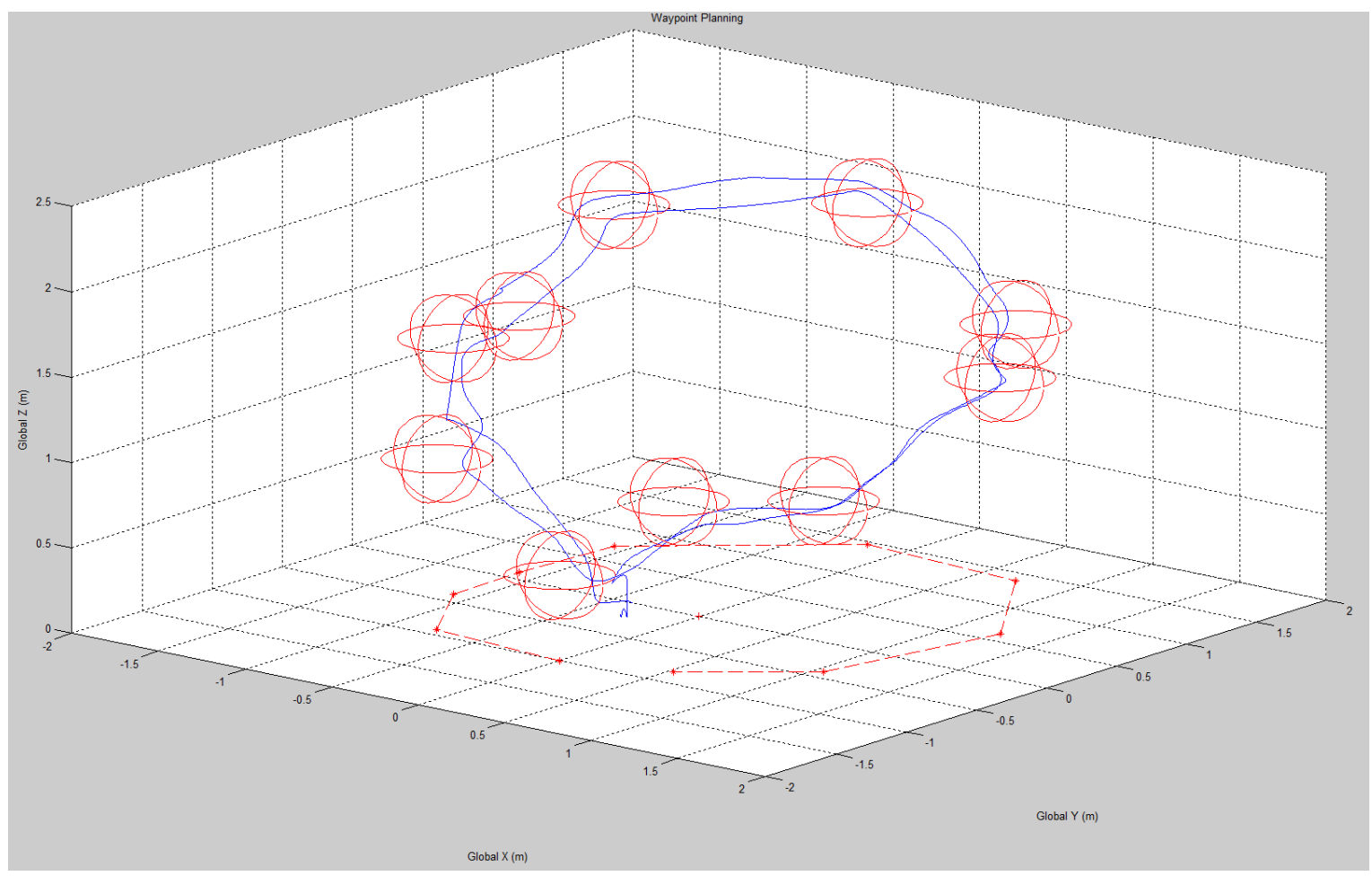

Figure 11: 3D Waypoint Visualization showing waypoints in red and the flight path in blue

This control scheme itself accepts the Vicon position data $(X, Y, Z)$ and angle values for roll, pitch, and yaw $(\varphi, \theta, \psi)$ and the current frame number as inputs at $50 \mathrm{~Hz}$, as well as the desired location of the quadrotor from Matlab (see Figure 12). The angles are converted from the global frame to the local frame using the direction cosine matrix shown above in Equation 1 since local angles are of most interest. These are represented by inputs 7-9 in Figure 12. Position is kept in the global frame since the quadrotor's position with respect to the testing area is most important, and is shown by inputs 4-6 in Figure 12. The desired positon is entered directly from Matlab as the series of waypoints described above as input 11. Position error in the global frame for each component is simply calculated as the desired position subtracted from the actual position, seen in Equation 13 below. 


$$
\begin{aligned}
& x_{\text {error }}=x_{\text {actual }}-x_{\text {desired }} \\
& y_{\text {error }}=y_{\text {actual }}-y_{\text {desired }} \\
& z_{\text {error }}=z_{\text {actual }}-z_{\text {desired }}
\end{aligned}
$$

The velocity is calculated from the position and current time step information directly from Vicon, and converted into the local frame as shown by Figure 12 inputs 1-3. The desired velocity components are calculated by setting a desired total velocity and multiplying that with the desired unit vector in each direction which is calculated from the position error. The unit vectors are reflected in the equations below.

$$
\begin{aligned}
& U_{x}=-\frac{x_{\text {error }}}{\sqrt{x_{\text {error }}^{2}+y_{\text {error }}^{2}+z_{\text {error }}^{2}}} \\
& U_{y}=-\frac{y_{\text {error }}}{\sqrt{x_{\text {error }}^{2}+y_{\text {error }}^{2}+z_{\text {error }}^{2}}} \\
& U_{z}=-\frac{z_{\text {error }}}{\sqrt{x_{\text {error }}^{2}+y_{\text {error }}^{2}+z_{\text {error }}^{2}}}
\end{aligned}
$$

The resulting desired velocity components are then found using the following equations using the method as described above.

$$
\begin{aligned}
& V_{x_{\text {desired }}}=V_{\text {total }_{\text {desired }}} * U_{x} \\
& V_{y_{\text {desired }}}=V_{\text {total }_{\text {desired }}} * U_{y} \\
& V_{z_{\text {desired }}}=V_{\text {total }_{\text {desired }}} * U_{z}
\end{aligned}
$$

These are shown in Figure 12 below by input 10 . The velocity error is then simply calculated by taking the difference between actual and desired velocity using the following equations. 


$$
\begin{aligned}
& V_{x_{\text {error }}}=V_{x_{\text {actual }}}-V_{x_{\text {desired }}} \\
& V_{y_{\text {error }}}=V_{y_{\text {actual }}}-V_{y_{\text {desired }}} \\
& V_{z_{\text {error }}}=V_{z_{\text {actual }}}-V_{z_{\text {desired }}}
\end{aligned}
$$

The desired yaw angle is simply set using a constant value shown by input 12 in Figure 12, but may easily be changed to be either the angle to the next waypoint or another object in the room defined by the Vicon system.

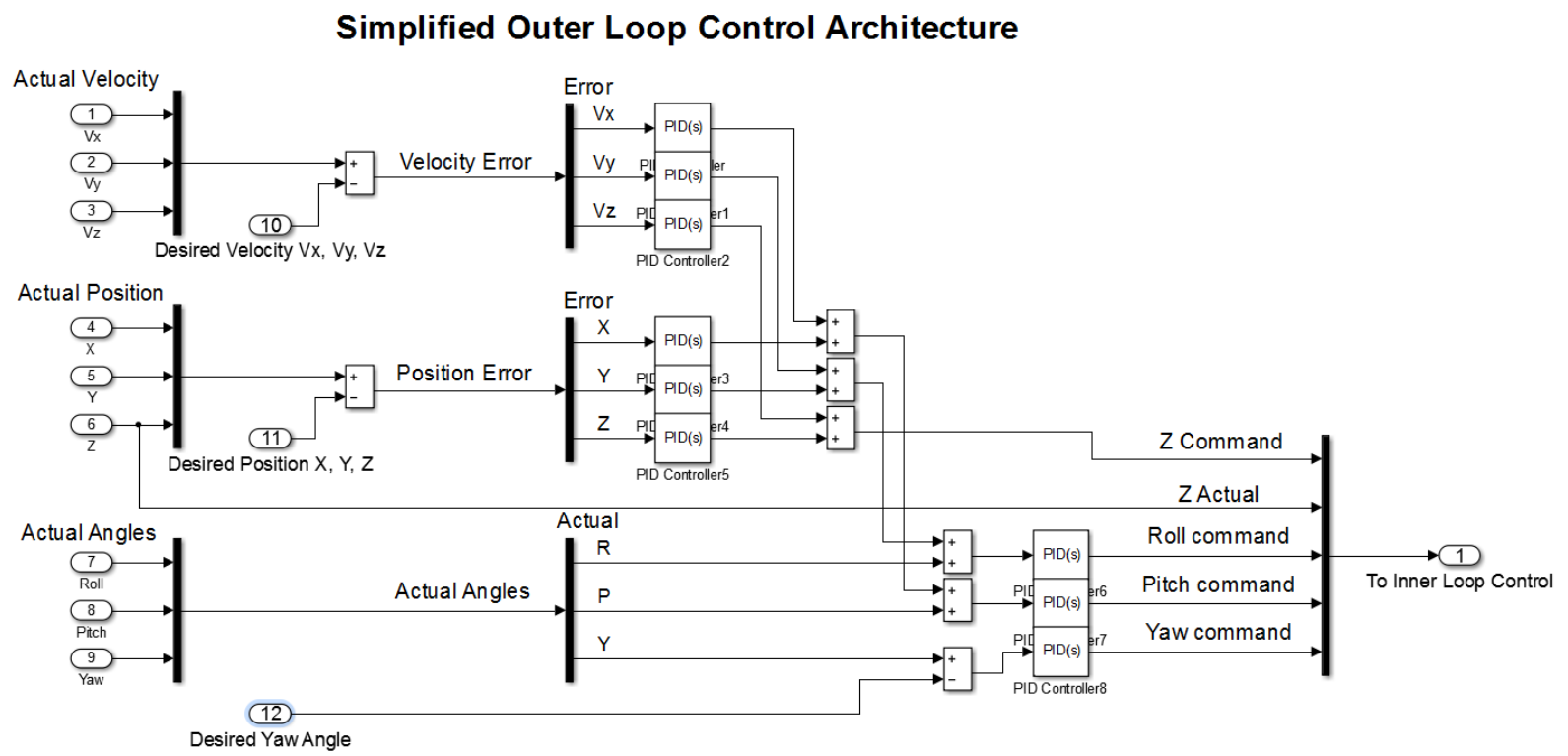

Figure 12: Simplified Outer Loop Control Scheme

The errors from position and velocity are then tuned using Proportional Integral (PI) gains which were tuned experimentally (see Table 2). These PI gains are then used in the following equation to manipulate the signal:

$$
u(t)=K_{p} e(t)+K_{i} \int e(t) d t
$$

Where $u(t)$ is the output, $K_{p}$ and $K_{i}$ are the proportional and integral gains, respectively, and $e(t)$ is the error signal. 
Table 2: PID gains for Outer Loop Control

\begin{tabular}{|c|c|c|c|c|}
\hline Gain \ Component & X & Y & Z \\
\hline \multicolumn{5}{|c|}{ Positon Error } \\
\hline Proportional & 10 & 10 & 750 \\
\hline Integral & 0 & 0 & 0 \\
\hline Proportional & \multicolumn{2}{|c|}{ Velocity Error } & \\
\hline Integral & 12 & 12 & 750 \\
\hline
\end{tabular}

The tuned error values are then combined, with the altitude error and the vertical velocity error combining to give the altitude $(\mathrm{Z})$ command. The error in the $\mathrm{X}$ direction and velocity error in the $\mathrm{X}$ direction are combined and used with the actual pitch angle to determine the pitch command, and the error in the $\mathrm{Y}$ direction and the velocity error in the $\mathrm{Y}$ direction are joined to be used with the roll angle, determining the roll command. The desired yaw angle is subtracted from the actual yaw angle to produce the yaw command. These commands are then tuned with the Proportional gains shown in Table 3 and then transmitted to the Inner Loop controller.

Table 3: Proportional gains for Roll, Pitch, and Yaw Commands

\begin{tabular}{|c|c|c|c|}
\hline Gain I Component & Roll & Pitch & Yaw \\
\hline Proportional & 60 & 60 & 200 \\
\hline
\end{tabular}

\section{Inner Loop Control}


An Inner Loop controller was coded for a NetBurner Core Module, which interfaces with the quadrotor's avionics board, with the goal of achieving stable flight with a controller that could be modified to suit a variety of desired uses. The first quadrotor was modified to use an avionics circuit board that was designed in-house to be used with the NetBurner and an IMU instead of the stock controller. The architecture of this Inner Loop controller essentially mimics the stock controller with $\mathrm{RC}$ communication and IMU feedback. The process of testing and refining the controller to stabilize the quadrotor using IMU feedback was carried out in phases through trial and error. First the roll axis was tested by restricting the quadrotor's movement by a simple tether system, and gains were tuned until desired performance was attained. Once stable on the roll axis, the pitch axis was tuned the same way. Finally, yaw was tuned using untethered RC flight so that performance was nearly identical to the original controller.

Additional capabilities were added to the Inner loop, starting with a motor kill switch to disable the motors in dangerous situations. A switch on the RC transmitter may be toggled, which signals to the NetBurner to immediately turn off all motors. Next, auto takeoff and landing sequences were coded into the controller to allow increased autonomy during flight. Another switch on the transmitter is monitored by the NetBurner, and when toggled it adds to the throttle value being sent to all motors. Also, the laser rangefinder was calibrated and integrated into the quadrotor's electronics. The data from the rangefinder is read by the NetBurner using analog to digital conversion. Finally, the avionics were connected to the XBee wireless modem to allow communication with the Outer Loop control scheme running on the ground station.

In the Inner Loop controller, commands are processed from the Outer Loop scheme, the quadrotor's IMU, and RC transmitter and interpreted into motor commands. As roll, pitch, and yaw commands from the Outer Loop enter, they are processed as shown below in equations 18-20. 


$$
\begin{gathered}
\phi_{\text {control }}=\left(\phi_{\text {command }}\right)+\left(K_{\phi}\right) *\left(e_{\dot{\phi}}\right) \\
\theta_{\text {control }}=\left(\theta_{\text {command }}\right)+\left(K_{\theta}\right) *\left(e_{\dot{\theta}}\right) \\
\psi_{\text {control }}=\left(\psi_{\text {command }}\right)+\left(K_{\psi}\right) *\left(e_{\dot{\psi}}\right)
\end{gathered}
$$

Where the control variable is $x_{\text {control }}$, the control command from the Outer Loop controller is $x_{\text {command }}$, the proportional gain is $K_{x}$, the IMU rate error is given by $e_{\dot{x}}$, and $\mathrm{x}$ is roll $(\phi)$, pitch $(\theta)$, or yaw $(\psi)$. The roll gain is 20 , pitch gain is 20 and the yaw gain is 30 . The IMU rate error is simply the rate gyro outputs from the IMU. These are denoted as errors since the quadrotor is desired to be stable with very little or no angular motion. These control variables are then fed into the following equations 21-24 along with the altitude $(Z)$ command from the Outer Loop and the pilot inputs from the RC transmitter.

$$
\begin{gathered}
M_{1}=T_{\text {control }}+T_{\text {pilot }}+\theta_{\text {control }}+\theta_{\text {pilot }}+\psi_{\text {control }}+\psi_{\text {pilot }} \\
-\phi_{\text {control }}-\phi_{\text {pilot }}+Z_{\text {command }} \\
M_{2}=T_{\text {control }}+T_{\text {pilot }}+\theta_{\text {control }}+\theta_{\text {pilot }}-\psi_{\text {control }}-\psi_{\text {pilot }} \\
+\phi_{\text {control }}+\phi_{\text {pilot }}+Z_{\text {command }} \\
M_{3}=T_{\text {control }}+T_{\text {pilot }}-\theta_{\text {control }}-\theta_{\text {pilot }}+\psi_{\text {control }}+\psi_{\text {pilot }} \\
+\phi_{\text {control }}+\phi_{\text {pilot }}+Z_{\text {command }} \\
M_{4}=T_{\text {control }}+T_{\text {pilot }}-\theta_{\text {control }}-\theta_{\text {pilot }}-\psi_{\text {control }}-\psi_{\text {pilot }} \\
-
\end{gathered}
$$


Where M1 through M4 are the commands to be sent to each motor indicated by their respective subscript indices. T is throttle, $\phi$ is roll, $\theta$ is pitch, $\psi$ is yaw, and $Z_{\text {command }}$ is the Outer Loop altitude control command. The subscript pilot refers to input from the RC transmitter, and the subscript control refers to the values given in equations 18-20 for roll, pitch, and yaw, except $T_{\text {control }}$ which is the throttle command given by the auto takeoff and landing routine.

\section{Planning and Control Algorithms}

1. D* Path Planning

In order for a quadrotor to travel from one location to another in a given environment, there are several prerequisites. First, the quadrotor must know where it is in the environment, where it needs to go, and if there are any known obstacles along the way. Using this information, a map may be constructed with a start point and a goal as well as the locations of the obstacles. In order to make such a map computer friendly, it may be discretized into an occupancy grid comprised of cells. Each of these cells represents a small area on the map and is given a value of zero or one, where zero indicates the cell is traversable and one means the cell is occupied by an obstacle. A greater number of smaller cells will allow for increased definition and therefore accuracy of the map, but is computationally intensive.

Once a map has been generated, the computer should have a reasonably accurate representation of the space the quadrotor will traverse. Next, it must decide the best route the quadrotor should take to reach its goal. Peter Corke's book Robotics, Vision, and Control [82] reviews several map based planning methods, and Corke has built the Robotics Toolbox for Matlab to accompany his book, which is a compilation of simple examples of a number of algorithms. The algorithms chosen for this research are the $D^{*}$ method and the Probabilistic Roadmap method, and the toolbox's code for these algorithms is used as a starting point. An example of a $D^{*}$ planned path is shown below, where the start point is on the bottom edge of the graph at coordinates $(30,1)$, and the goal is at $(80,80)$ (see Figure 13$)$. 


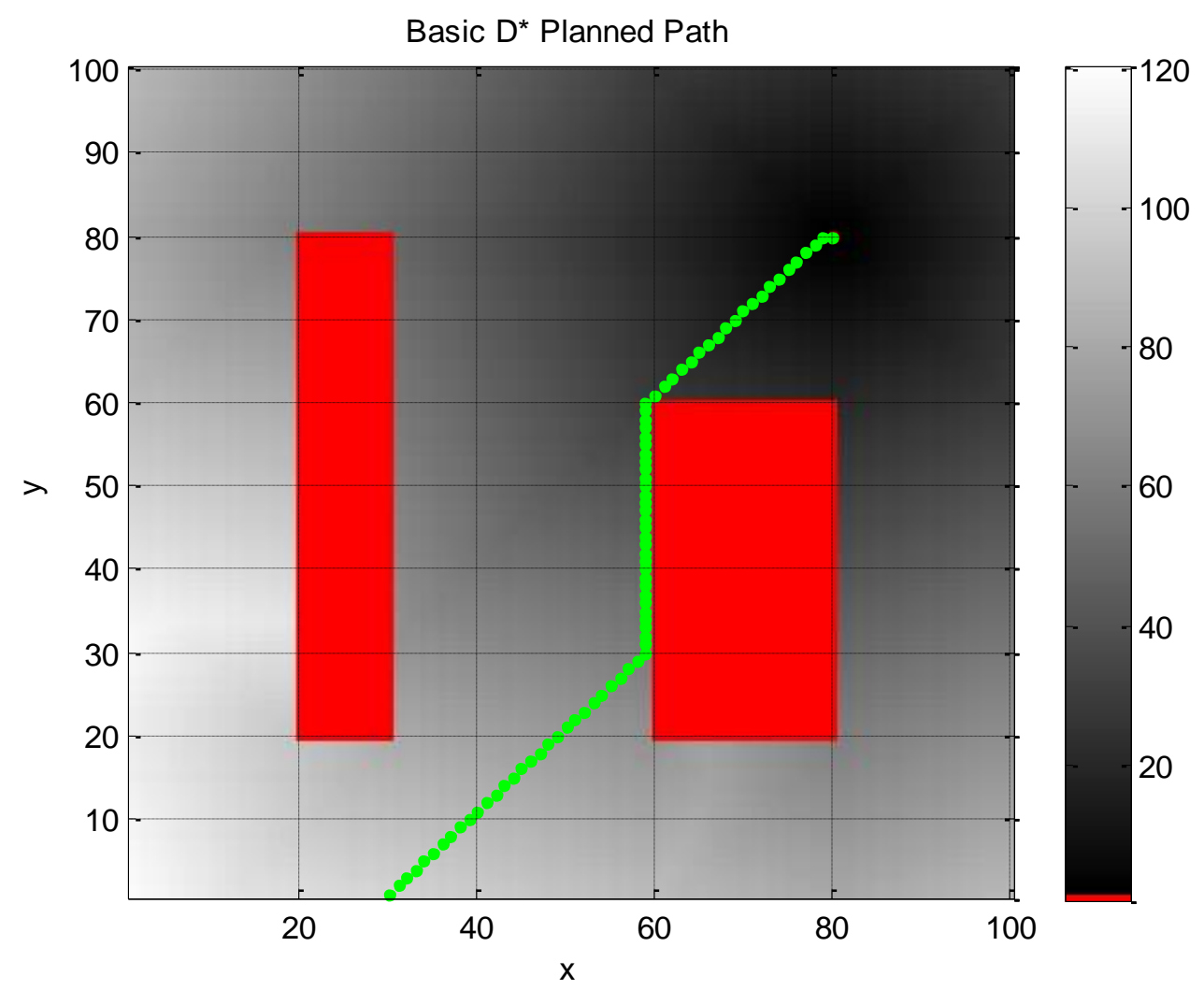

Figure 13: Corke's D* Simulator Costmap and Planned Path (obstacles shown in red)

With the $D^{*}$ method, a costmap is generated where each node in the map is assigned a cost from zero to infinity based upon distance from the goal coordinate, with lower numbers meaning lower cost to reach the goal. The lowest cost is the goal coordinate, with all neighboring nodes having the next lowest cost, and so on. Locations with obstacles have infinite cost. The planner then works backwards from the goal to the start location to find the path that follows the lowest costs. The $\mathrm{D}^{*}$ algorithm allows for costmap changes, so that if an obstacle or more "difficult" terrain, are encountered they can be added to the costmap. Once the costmap has been adjusted, a replan must occur and a new path must be taken. This replan is less computationally intensive than a global replan, as it just modifies existing information.

Before the algorithm can be discussed in depth, there is a large amount of terminology to be defined. First, the nodes on the map are referred to as "states" with X being a generic state. They are connected by "directional arcs" which have costs associated with them. G is 
defined as the goal state. All states are defined with a "backpointer" to the next state $Y$ which is given by $b(X)=Y$. These backpointers are how the algorithm shows a path. The cost of moving from $\mathrm{Y}$ to $\mathrm{X}$ is given by $\mathrm{c}(\mathrm{X}, \mathrm{Y})$ which is the "arc cost" function, which is used to generate the costmap discussed above. If the cost function is defined for either $c(Y, X)$ or $c(X, Y)$ then $X$ and $\mathrm{Y}$ are said to be "neighbors". There is a grouping of states called the "openlist" which allows information to be passed about arc cost function changes and calculates path costs to other states. All states have an associated "tag" $t(X)$ which is assigned to be NEW if $X$ hasn't been in the openlist before, OPEN if it is in it right now, and CLOSED if it is not on the openlist anymore. Also along with each state is kept a (theoretically optimal) sum of the arc costs from $X$ to $G$, given by the "path cost" function $\mathrm{h}(\mathrm{G}, \mathrm{X})$. For states on the openlist, there is a "key function" which is the minimum of $h(G, X)$ before any changes are made and all assumed values by $h(G$, $\mathrm{X}$ ) since it has been on the openlist. It says whether $\mathrm{X}$ is a "raise" state, meaning it is associated with path cost increases, or a "lower" state, meaning it is associated with path cost reductions or a new path. This information is given to neighboring states when $\mathrm{X}$ is taken out of the openlist, which are then placed on the openlist themselves. The value $k_{\min }$ is the minimum of the key function, meaning that path costs less than or equal to $k_{\min }$ are optimal and greater path costs may not be optimal. Another important value, $k_{\text {old }}$, is defined as $k_{\min }$ before the latest removal of a state from the openlist. The ordering of states is called a "sequence" which is a path of backpointers from $X_{1}$ to $X_{N}$. It is monotonic if the tag is CLOSED and $h\left(G, X_{i}\right)<h\left(G, X_{i+1}\right)$ or if the tag is OPEN and $k\left(G, X_{i}\right)<h\left(G, X_{i+1}\right)$ for $1<i<N$. The algorithm keeps such a sequence for each state that is or was on the openlist, which denotes decreasing current or lower-bounded path costs. The primary functions are ProcessState, which looks for the best path costs to the goal, and ModifyCost, which changes the cost function and adds states to the openlist.

The algorithm is initialized by placing $G$ on the openlist, $h(G)$ is zero, and $t$ is set to NEW for all states. Then the algorithm begins by calling the function ProcessState until the state $\mathrm{X}$ is removed from the openlist or a value of -1 is returned. The path given by the backpointers is 
then followed until the robot either reaches the goal or detects an obstacle in its path. If the latter occurs the ModifyCost function is used to change the cost function and adds the states whose cost has changed to the openlist. Pseudocode for the algorithm is shown in Figure 14 [59]. 


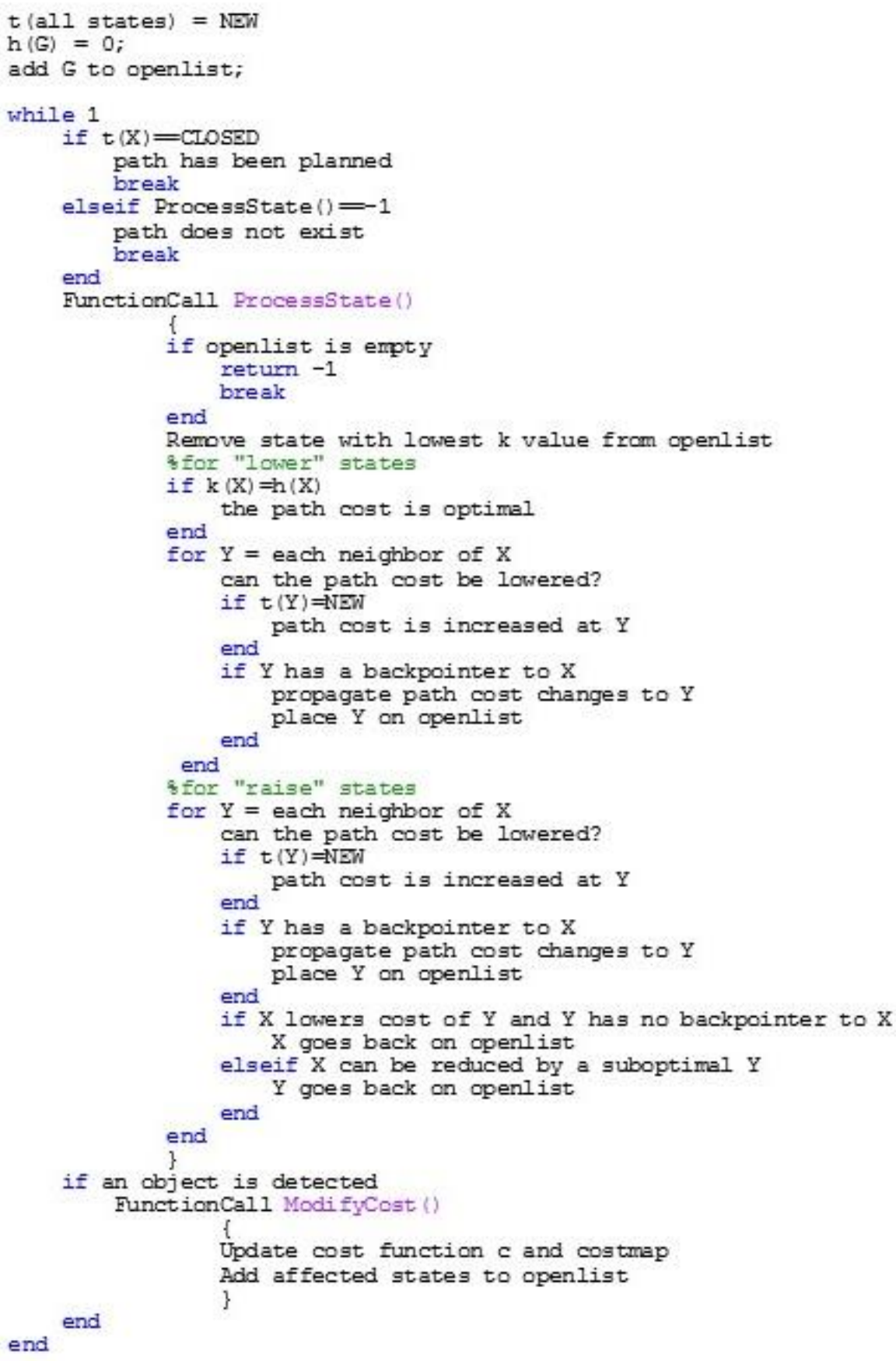

Figure 14: $D^{*}$ Pseudocode

Using this knowledge, a full redesign is made to the structure of the Corke's toolbox D* algorithm for several reasons. First is the fact that Corke's robot is assumed to occupy a single 
cell in the occupancy grid at any given point. This would be very difficult to achieve with a full sized quadrotor in a small area as the map resolution would suffer greatly. Therefore, in this application the resolution was increased to allow for a better map, and as a result the robot takes up much more than one cell. As an unwanted byproduct of this change, the algorithm will still cut corners very closely which would make the quadrotor hit the obstacles it is supposed to avoid. Therefore another change post-processes all obstacles that are placed in the map and finds their corners. A buffer zone is then created at forty-five degree angles from the corners before the path planning algorithm is implemented, so that the planner will not approach corners as close as it would before. The map was set to be a 100x100 grid and the buffer zones were somewhat arbitrarily set to 8 units long, which is sufficient to keep the quadrotor away from the obstacles when the $100 \times 100$ roadmap is scaled into the testing area's $4 \times 4 \mathrm{~m}$ space.

Once the path has been planned as a series of $x$ and $y$ points, a simulator displays the path on the map. In reality it would be quite difficult to use these points in control of the quadrotor, as it would need to reach each point exactly in 3D space in order to move on to the next point. This would require perfection in control through potentially hundreds of successive points. In order to ease these restrictions, two modifications were made. The original path is post-processed in Matlab to determine "critical points" in the path. These critical points are defined as points where the path turns suddenly. The critical points are then defined as waypoints for the quadrotor, greatly reducing the number of points it needs to follow. In addition, in the outer loop control algorithm only the next waypoint is given as the desired location. As described above in Section III Part C Number 1, the quadrotor is deemed to have reached the waypoints when it enters a half meter diameter sphere around the point, and the next waypoint on the list is then listed as the desired waypoint. This approach using a spherical waypoint instead of an exact point in 3D space allows for a less exact control algorithm while still ensuring a measure of precision (see Figure 15Error! Reference source not found.). 


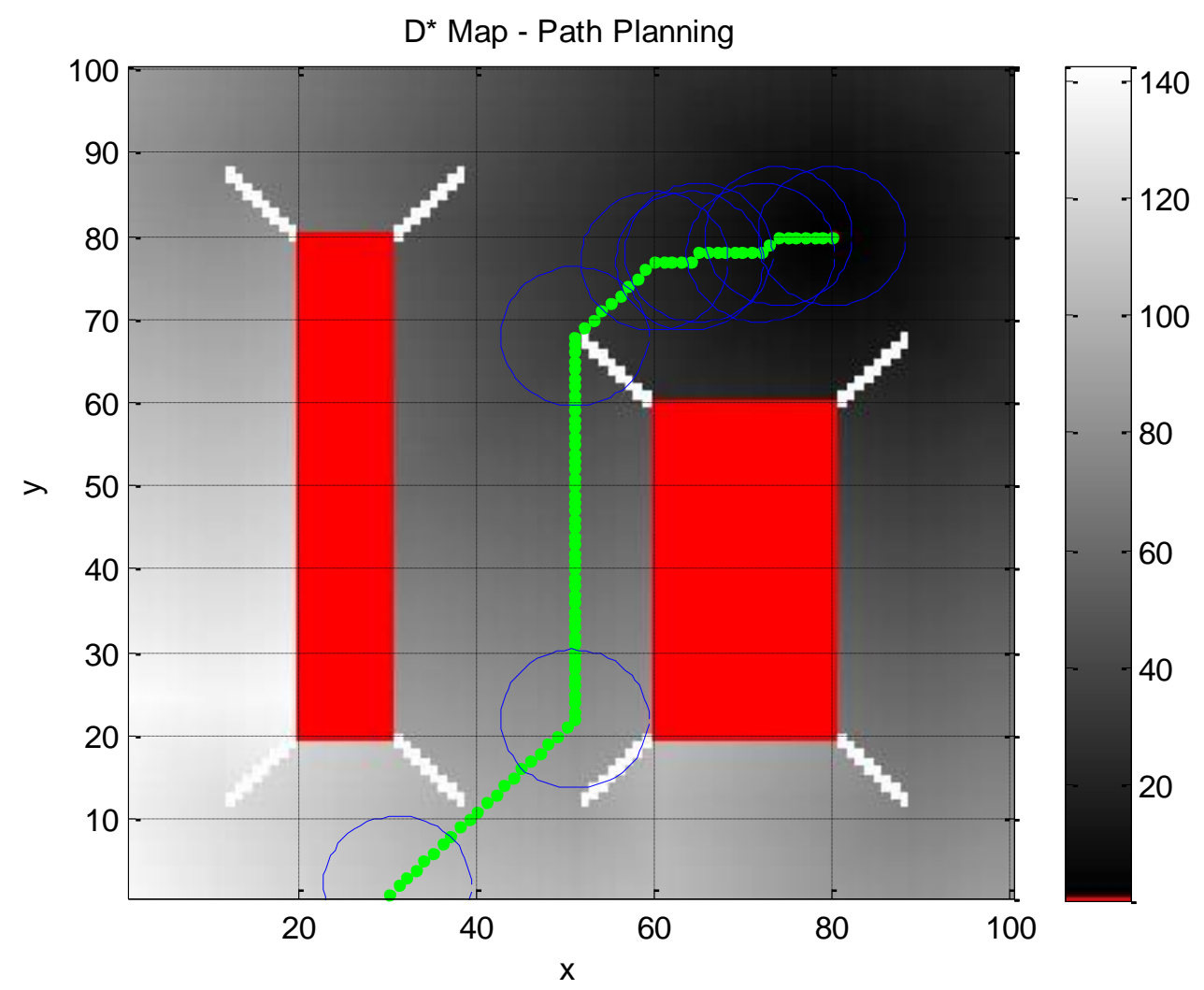

Figure 15: Redesigned D* Algorithm (waypoints shown in blue and buffer zones in white)

This algorithm is originally written for a simple Matlab demonstration, making coding the described redesign rather straightforward. This Matlab script is fine for planning purposes before a flight, and so the demonstration altered as described above to output waypoints and initialize variables to a Simulink model as well as display the planned path over the map.

However, this algorithm also needs to be executed in the Simulink Outer Loop control scheme in order to be used with the Vicon Motion Capture system for real-time replanning, therefore it had to be heavily modified again to be compatible with Simulink. The original Matlab algorithm uses a class hierarchy in order to call specific functions and their properties when needed, and changes variable sizes frequently. Neither classes nor variable size matrices are supported in Simulink, therefore the entire algorithm had to be re-written to accommodate these limitations. This was done by putting the entire $D^{*}$ algorithm into a single Matlab function block in Simulink. This block's main function initially has as inputs all of the class properties from the 
Matlab planner and calls all necessary sub-functions which are listed below the main function. The properties are then each passed into and then out of all subsequent function calls so that they may be used or modified as needed. The main function then outputs the final values of these properties, which are fed back into the function block as inputs for the next time step. All properties and other matrices are initialized to a fixed size and kept that size throughout the entire function block to ensure compatibility.

\section{Probabilistic Roadmap Method}

The other map based planning algorithm is the Probabilistic Roadmap method, which was tested to compare with $D^{*}$. This method uses an occupancy grid roadmap with obstacles defined as having a value of one and traversable areas having a value of zero. There are two phases to this method, the learning phase and the query phase. In the learning phase, the algorithm chooses a set number of randomly generated nodes, or configurations of the robot, that do not lie in the obstacles. It then iterates through each node and assigns an edge between it and all neighboring nodes that are closer than a certain threshold to denote that they are connected, meaning the robot may travel along the edge. This is all completed without needing the start and goal nodes. Once all nodes and edges are assigned, the query phase begins. The start and goal points are defined, the algorithm connects them to the closest nodes. The planner is asked to choose a path from the closest node to the start point to the closest node to the end point. There is no built-in replanning function similar to $D^{*}$. Instead, the occupancy grid must be modified and a global replan must occur, choosing new points and edges around the obstacles. Pseudocode for the algorithm is shown in Figure 16 [69]. 


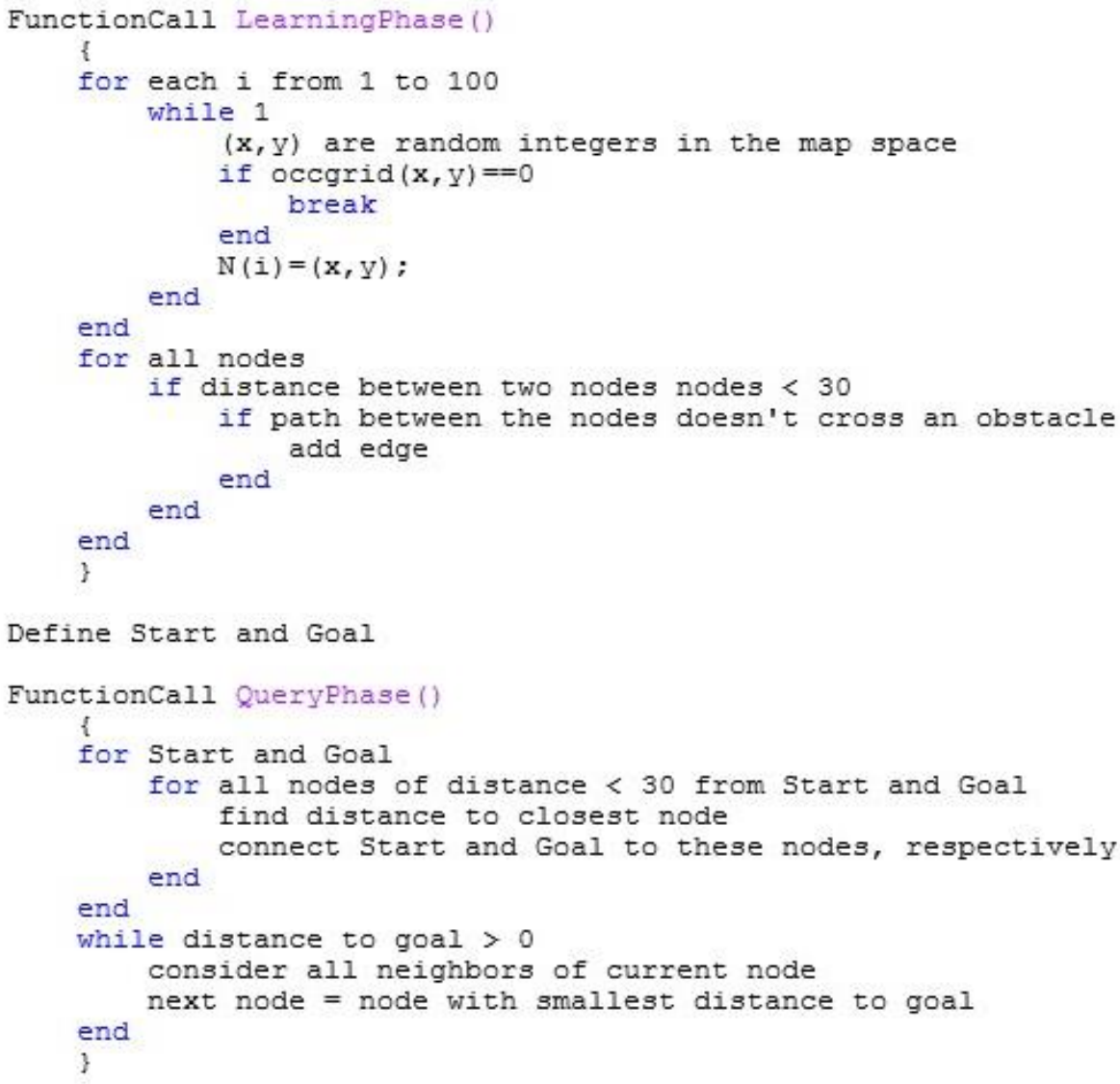

Figure 16: PRM Pseudocode 


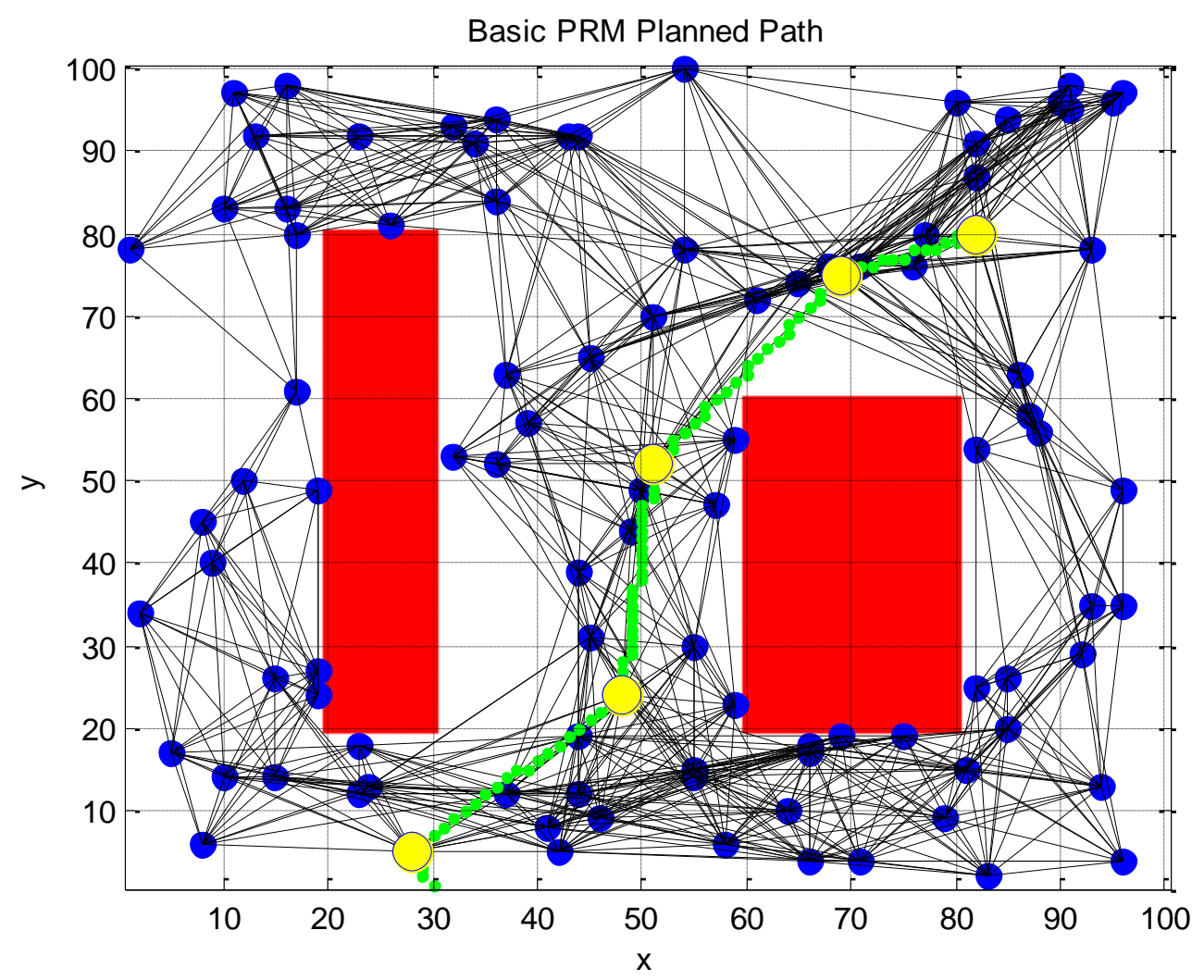

Figure 17: PRM Planned Path

For this specific application, once again the map is set to be a $100 \times 100$ grid. Node selection was changed to take into account an area around all of the obstacles as a buffer zone. The buffer zone is to ensure none of the points would be "too close" to the obstacles for the quadrotor to traverse by them, and was chosen to be the eight nodes in all directions around the obstacles. One hundred nodes were used, and edges were only assigned between nodes that were closer than thirty units. Generally this achieves adequate results, however the path may sometimes seem to be somewhat arbitrary or indirect due to the random nature of the node selection. However, instead of needing a post-processed path to choose the waypoints, the planner was simply modified to output the nodes that were chosen, shown in yellow in Figure 17 above. Note the start and end nodes are again $(30,1)$ and $(80,80)$, respectively. 


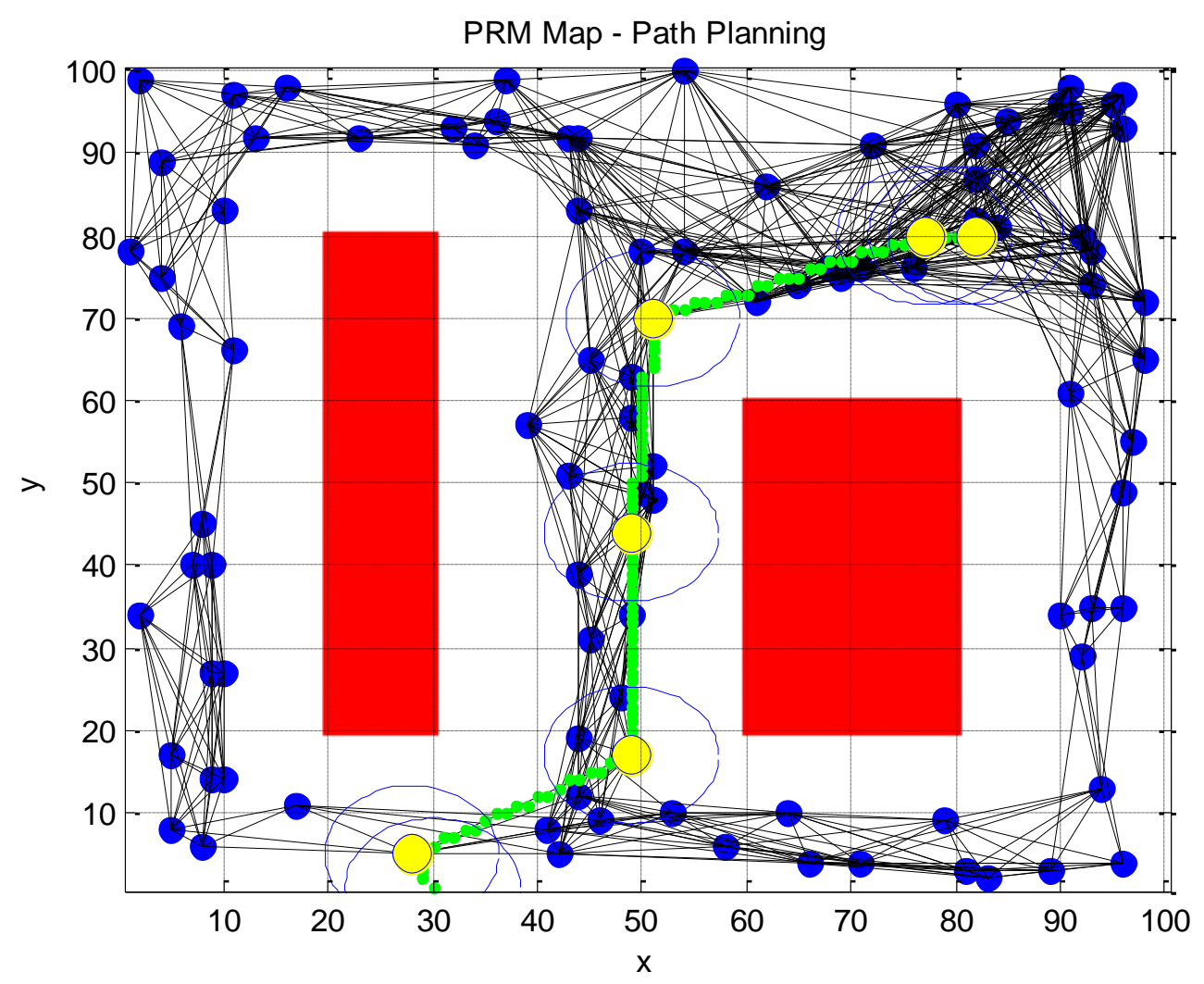

Figure 18: Modified PRM algorithm

A Matlab script planner was constructed to output the initial waypoints in the same way as the $D^{*}$ function. Next, the PRM function was modified for use in Simulink in exactly the same way as the $D^{*}$ function, with all properties from the Matlab planner's classes were inputs to the Simulink PRM replanner function. These properties are treated exactly the same as for the $D^{*}$ planner by being passed through all function calls.

Though this algorithm is intuitive, the main drawback of this planning algorithm is that, given the right circumstances it might not find a navigation solution. This becomes obvious when the map is changed to only allow navigation through a very tight passageway with the same start and goal points, as shown below in Figure 19. 


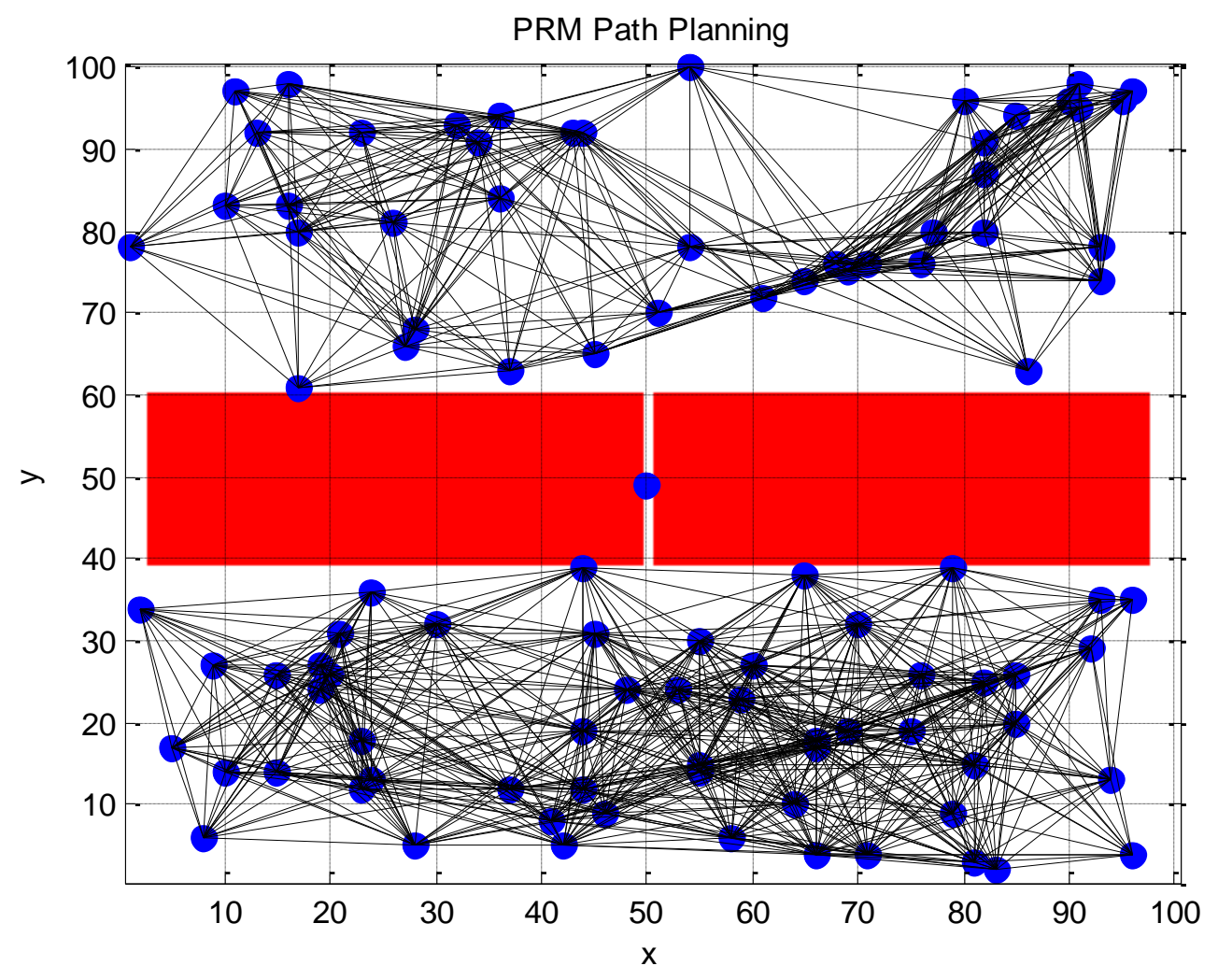

Figure 19: PRM Path Planner failure

In this extreme example, the PRM planner generates the correct number of points, one even in the middle of the passageway. However, there are no nearby nodes that can connect without passing through the obstacles, and therefore the planner will fail and generate an error. This configuration is not a problem for the $\mathrm{D}^{*}$ algorithm, as shown in Figure 20 below. 


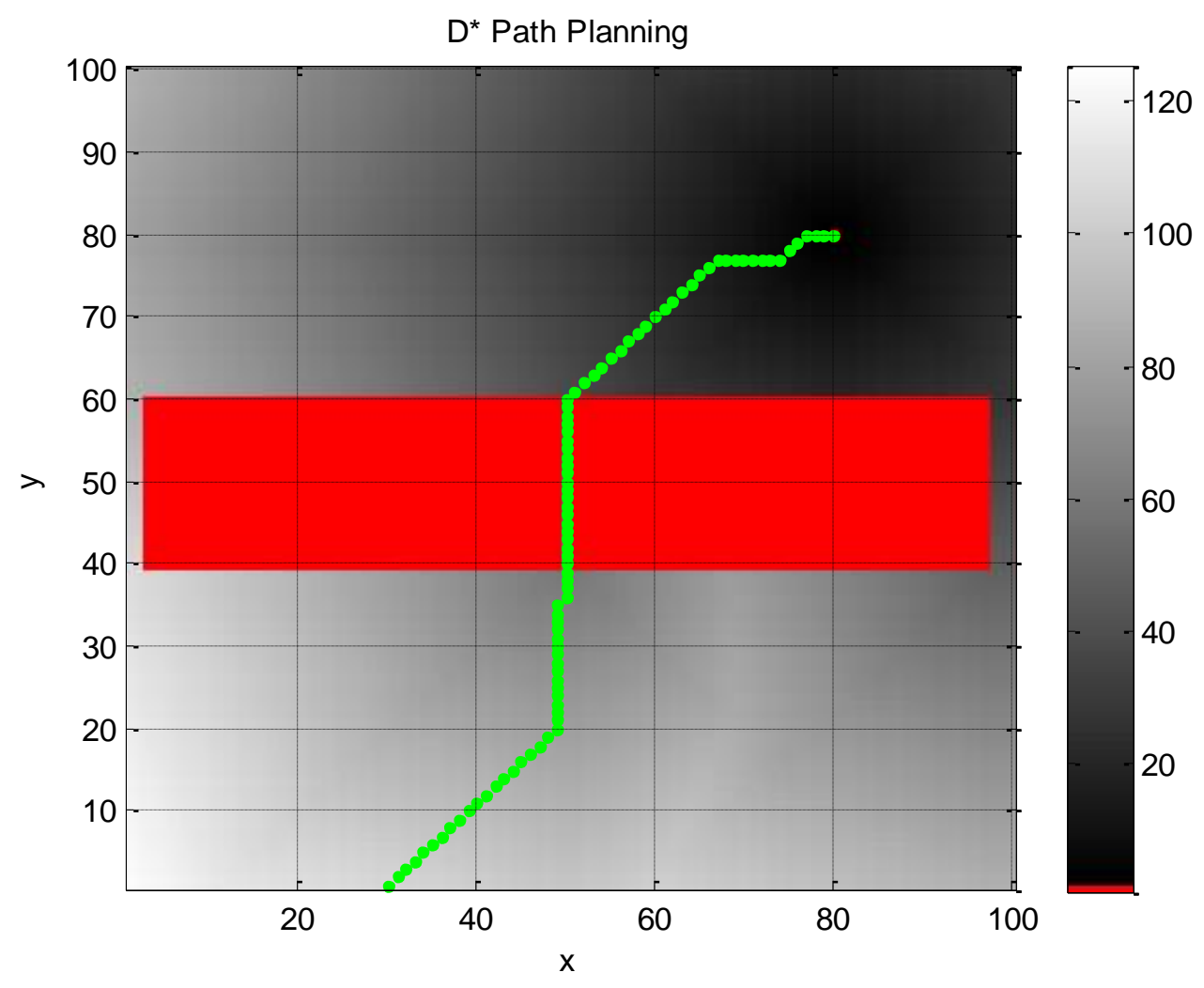

Figure 20: D* Path planning success

\section{E. Systems Integration}

A system relying on this amount of diverse software systems and the communication between them needs a high level of systems integration to ensure smooth functionality and consistent results. The Vicon Tracker system is connected to the Outer Loop control using Quanser's QUARC software toolbox for Simulink. This integrates seamlessly into the Outer Loop framework by providing an output block that sends all position and angle information to the scheme. On the output side of the Outer Loop, the control commands are combined into a packet to be sent to the quadrotor serially using XBee wireless modules. The inner loop controller is modified to accept the control commands in addition to the other data it was already collecting. The Vicon/Tracker system (see Figure 21) then offers closed loop position feedback to allow autonomous indoor flight with high precision. 


\section{Vicon/Tracker}

\section{Outer Loop}

\section{XBees}

Inner Loop

Figure 21: Quadrotor Data Flow

In order to tell the Outer Loop Control when the laser rangefinder detects an obstacle, the Inner Loop uses the XBee serial link to send back a simple 0 or 1 , where 0 means no obstacles are detected within a $0.75 \mathrm{~m}$ range, and 1 means an obstacle is less than $0.75 \mathrm{~m}$ away. This data is then read on the Outer Loop scheme, and when the value received is a 1, the scheme switches from the planned path to replan mode for the desired planner. At this point, the quadrotor's current position is stored as a new waypoint and told to hover there until path planning is completed. The yaw angle is then taken into account, and the obstruction location is found by the following equations.

$$
\begin{aligned}
& \text { obstruction }_{x}=x_{\text {hover }}+0.75 * \cos (\psi) \\
& \text { obstruction }_{y}=y_{\text {hover }}+0.75 * \sin (\psi)
\end{aligned}
$$

Where $x_{\text {hover }}$ and $\mathrm{y}_{\text {hover }}$ are the position the quadrotor is hovering at, 0.75 is the trigger distance in meters, and $\psi$ is the yaw angle. The obstacle is then extended out on either side from this point in order to block the passageway, and added to the map for replanning. Once replanning is complete in a single Simulink time step, the scheme then will use the replanned waypoints for the rest of the flight. 


\section{Testing}

\section{A. Simulation}

Before flight testing, both path planning algorithms were tested in Matlab to see how they would perform, to provide a stepping stone to real-life implementation, and to ensure replanning was viable. The same map to be used for flight testing is used here as well as the same start and goal nodes, $(30,1)$ and $(80,80)$.

To begin, the planner is run to plan the initial path from start to the goal. The map is processed and a costmap is built. The path maneuvers around the obstacles from start to finish, and the waypoints are found for this path. However, an "unmapped" obstacle is inserted between the two obstacles using the ModifyCost function, completely blocking the center path (see Figure 22).

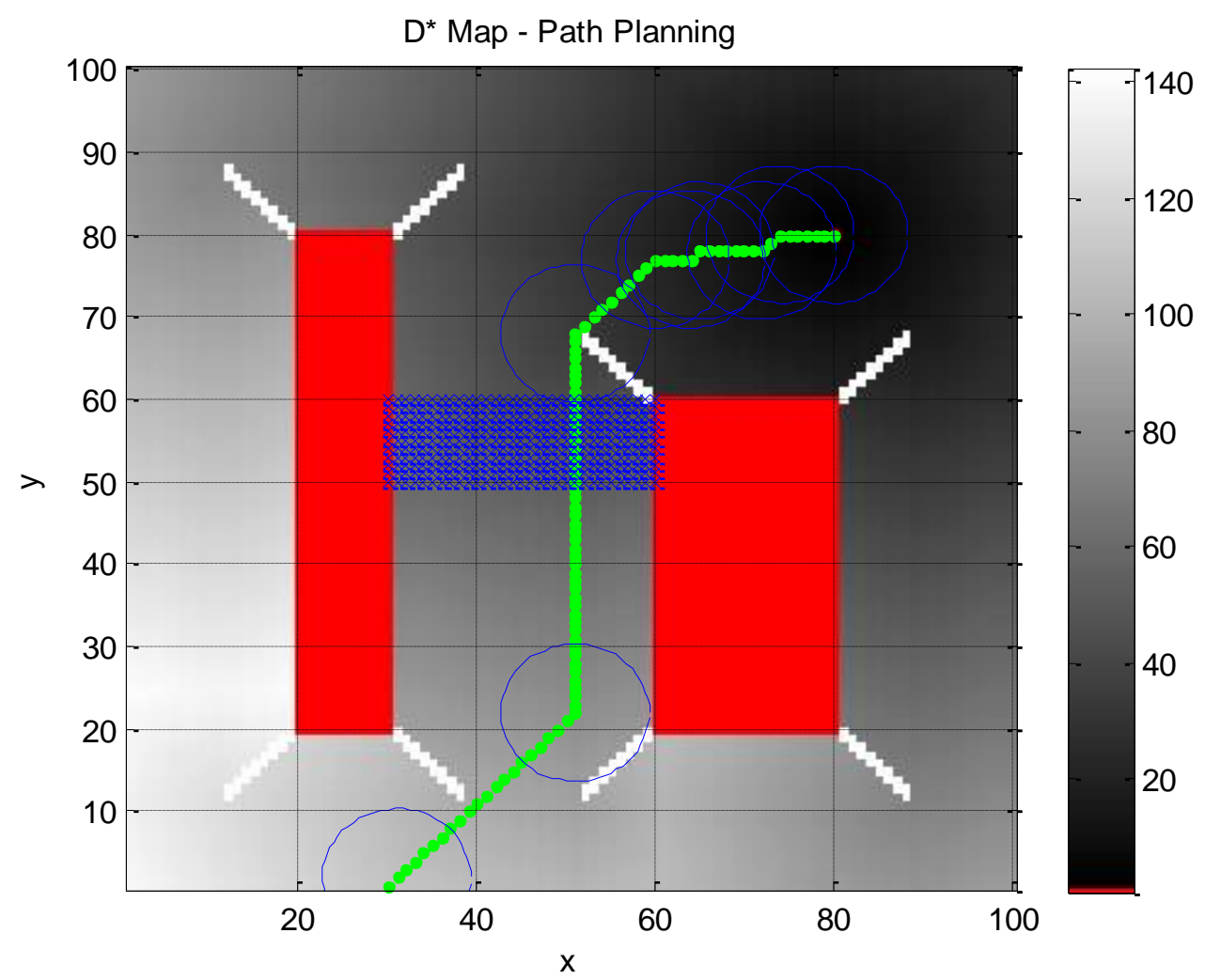

Figure 22: D* Simulation - Planned Path and Unmapped Obstacle 
The simulator is then asked to decide if it will cross the unmapped obstacle (shown in blue), and if so to find out where. It stores this crossing point as the start location for the replan as well as the location of the obstacle. It is then asked to find a new path to the same goal location with the new information about the obstacle, starting from the crossing point.

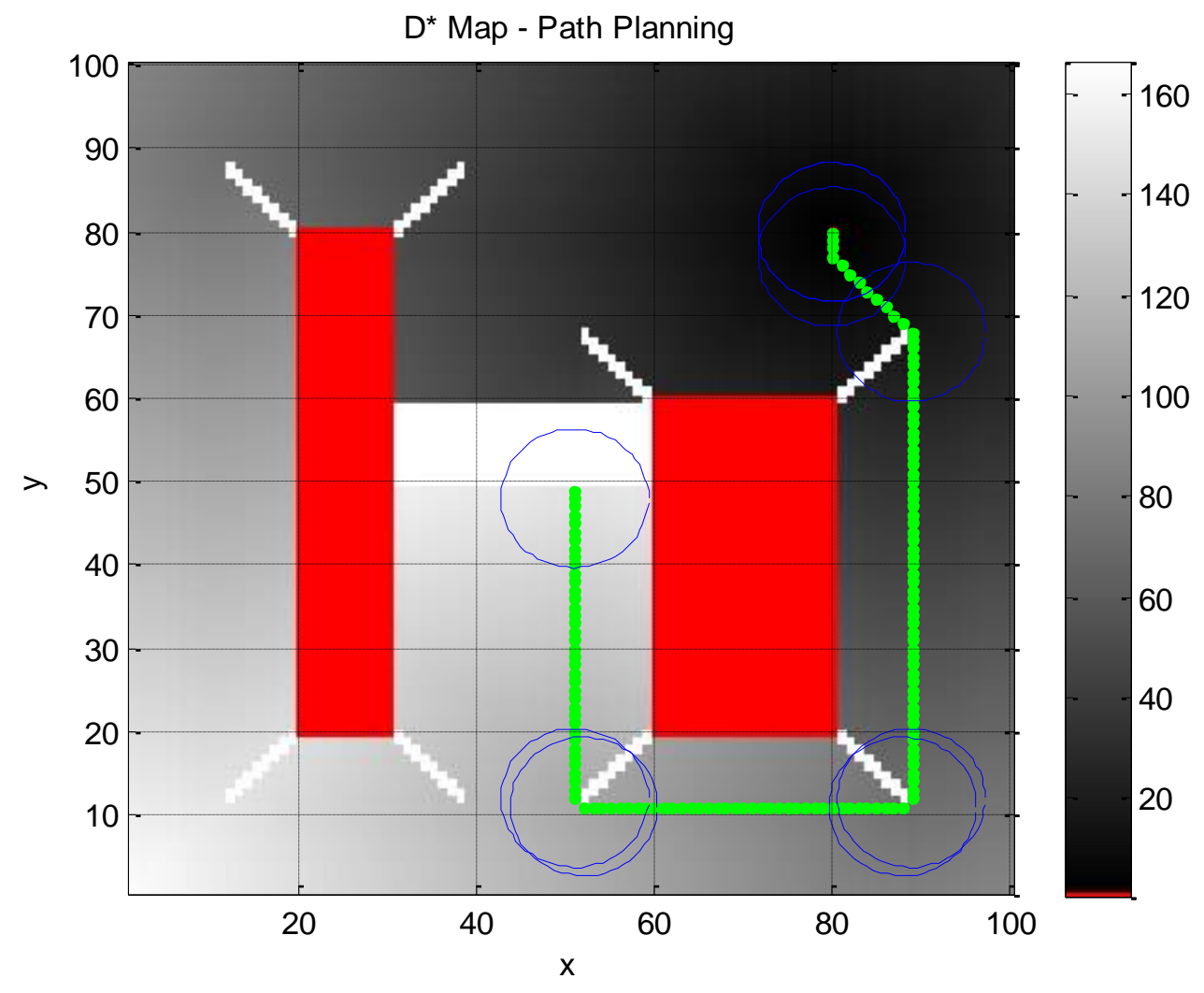

Figure 23: D* Simulation - Replanned Path

This replanned path is reflected in Figure 23 shown above with the new obstacle added to the costmap as infinite cost. The incremental replan then finds the best path to the goal around the obstacles, concluding the $\mathrm{D}^{*}$ path planning simulation. 


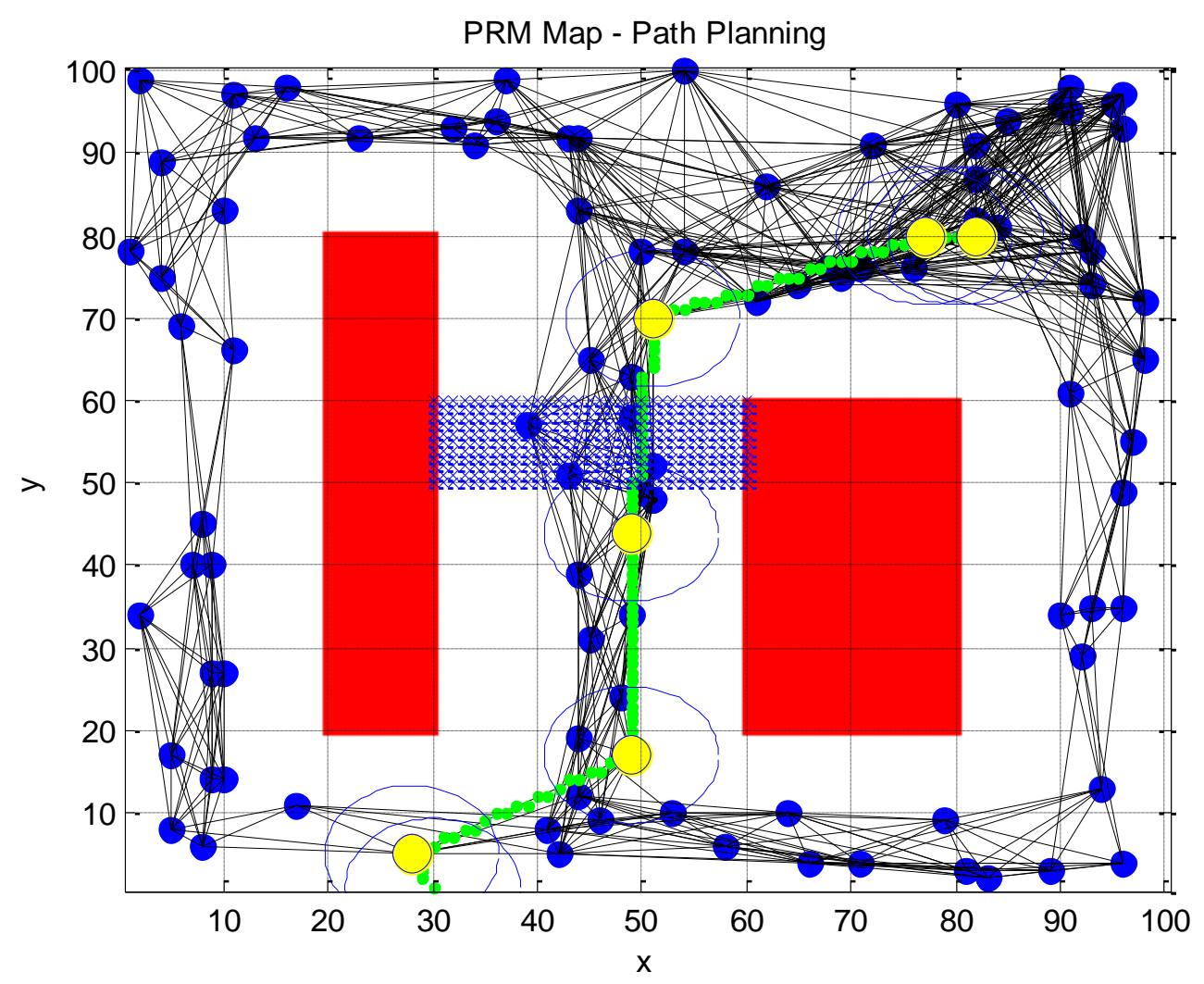

Figure 24: PRM Simulation - Planned Path with Unmapped Obstacle

The Probabilistic Roadmap method simulation seeks to achieve the same results on the same map. Using the same starting and ending locations the PRM planner selects nodes and edges around the obstacles and their buffer zones. A path is then chosen through the nodes from the start to goal, and these nodes are used as waypoints. However, an unmapped obstacle is inserted in the same location as the $D^{*}$ planner, which completely blocks the center path (see Figure 24).

The new obstacle is stored in the planner's occupancy grid. The planner is then asked to decide whether the path will cross this new obstacle, and if so then to find and store the crossing point. It is asked to replan a path from the crossing point to the goal location, taking into account the new obstacle. 


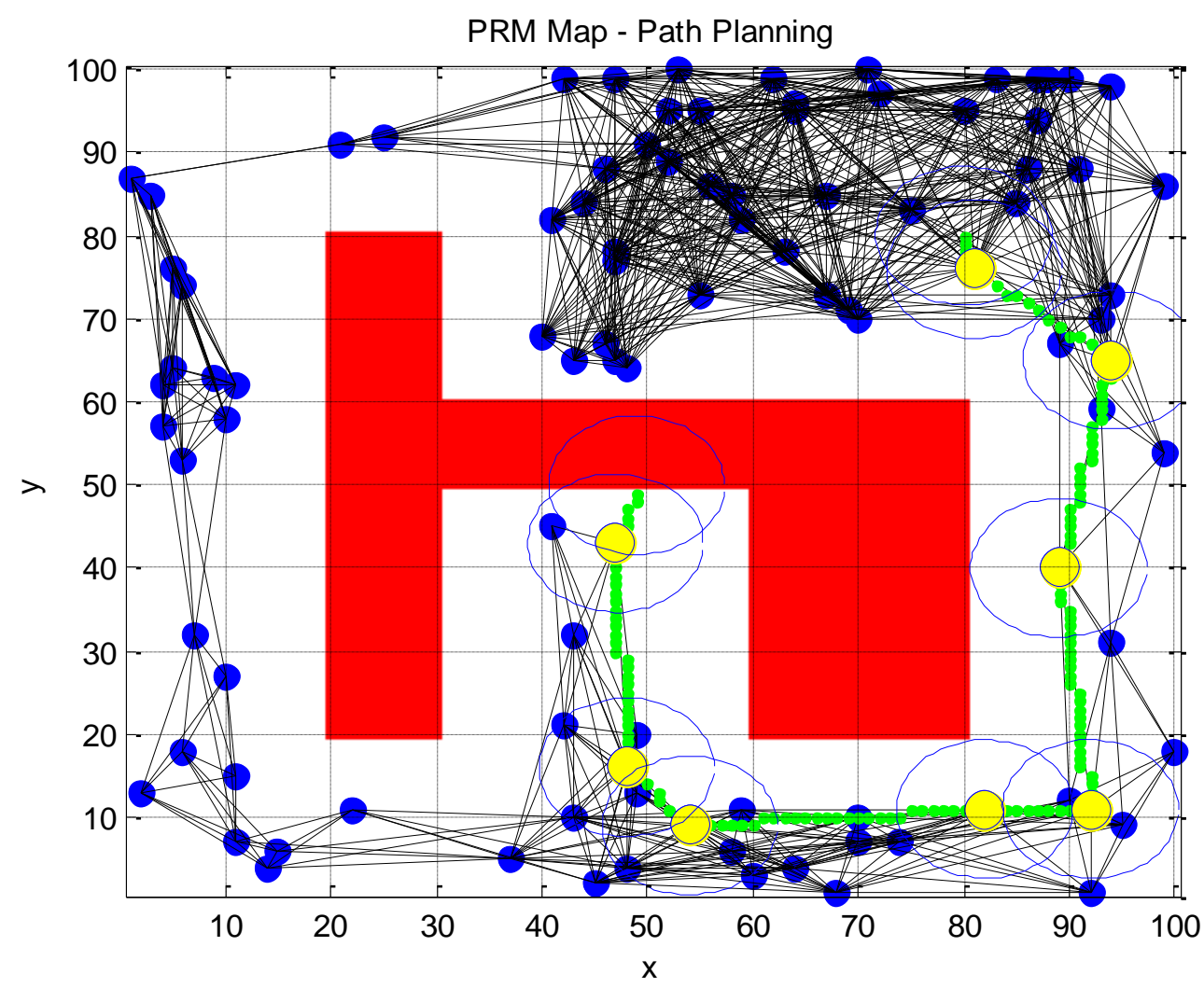

Figure 25: PRM Simulation - Replanned Path

The replanned path is shown above after new node and path selection, as well as waypoint extraction. This path successfully navigates around the obstacles, finding a path to the goal node. This completes the PRM replanning simulation.

\section{B. Flight Testing}

During flight testing, several simplifying assumptions were made based on the knowledge of the quadrotor's capabilities in order to ensure the safety of the quadrotor and everyone present. First, the quadrotor was set to a constant yaw angle so that if would face only one direction instead of the next waypoint, since the quadrotor has the potential to yaw wildly if it is close to the waypoint or when that waypoint changes abruptly. Along with this, object detection will occur only directly in from of the quadrotor as there is only one sensor being used for replanning. Replanning itself is only in two dimensions ( $\mathrm{X}$ and $\mathrm{Y}$ ) for simplicity, and altitude is 
held constant during flight. In addition, there are no actual physical obstacles in the testing area during flight in order to reduce risk of crash or injury to bystanders. Also, replanning will only occur once per flight, and the detected object will be assumed to block the entirety of the path between the two mapped obstacles.

During test flights, a safety pilot controls an $\mathrm{RC}$ transmitter communicating with an $\mathrm{RC}$ receiver on the quadrotor's autopilot board. The pilot may manually supersede autonomous flight if needed to ensure that the quadrotor will not do anything undesirable or unsafe. Another person monitors the Outer Loop Simulink control scheme on the ground station computer, and may alternate the quadrotor's flight mode between hover and execution of waypoint following. A third person wearing a helmet and safety goggles maneuvers an obstacle during flight for the quadrotor to detect.

Before $D^{*}$ flight, the Matlab $D^{*}$ path planner is run to initialize all parameters for the Simulink Outer Loop scheme. It uses the same map as well as start and goal nodes as the simulation, plans waypoints from the start to the goal, and then adds preprogrammed intermediate waypoints around the outside of the leftmost obstacle to allow the quadrotor to return to start, and in this way continually repeat the planned waypoints (see Figure 26). Note that the figure below is shown in the room's $4 \times 4$ meter space with the origin in the center and not the planner's $100 \times 100$ space with the origin in the bottom left corner. Also note the axes are different from normal convention, as the vertical axis is the Inertial Frame's X-axis, and the horizontal axis is the Inertial Frame's Y-axis, and that the values shown on the $\mathrm{Y}$-axis are negative. While somewhat confusing, this is to allow consistent views of the objects and waypoints. Therefore, using the point $(50,50)$ as the origin of this space, the starting point of the algorithm is converted from $(30,1)$ in the planner's coordinates to $(-1.5,0.5)$ in the Inertial Frame's coordinates. 


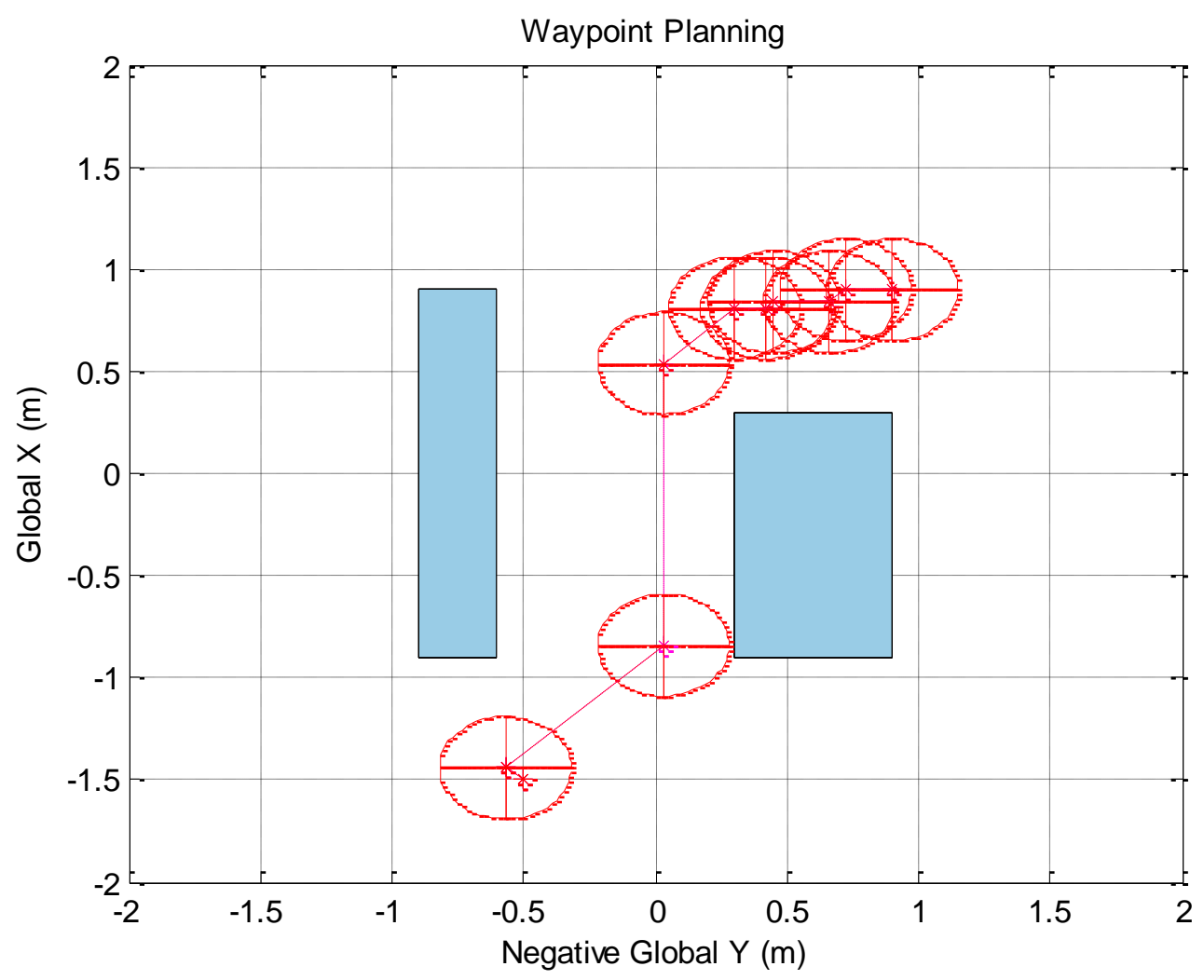

Figure 26: Pre-flight Visualization of map and planned path

This Outer loop scheme is then run inside the Simulink model. Once the scheme is running, the quadrotor is powered on with motors disabled. The communication LED is monitored, and once it is apparent that the ground station and quadrotor have established communication the motors are enabled by the safety pilot's RC transmitter. He then engages the auto takeoff routine, and the motors spin up until the quadrotor lifts off and reaches its desired hover altitude of $1 \mathrm{~m}$. Once hovering, the person controlling the ground station computer toggles the flight mode so that the quadrotor begins flying from waypoint to waypoint (see Figure 27). The quadrotor successfully flies from the start to goal node and then executes the intermediate waypoints to allow it to return to the start node and repeat. On the second iteration of these waypoints, the third person moves an obstacle directly in front of the quadrotor's flight path between the two mapped obstacles. When the quadrotor's laser rangefinder detects the obstacle at $0.75 \mathrm{~m}$ away, it triggers the replanning portion of the Outer Loop scheme. In one 
time step of the Simulink scheme, the quadrotor's yaw angle is taken into account along with the detection distance of $0.75 \mathrm{~m}$ to determine the exact location of the detected obstacle. This location is then expanded in both directions perpendicular to the quadrotor's flight path so as to create an imaginary wall. These coordinates are used to update the cost map of the $\mathrm{D}^{*}$ algorithm, and then a replanned path is calculated, and a set of new waypoints is found. These waypoints replace the initially planned waypoints and the quadrotor begins to execute this replanned path on the next time step. The quadrotor follows the new path around the obstacles until it reaches its destination at the goal node. Once at the goal, the quadrotor hovers until the safety pilot initiates the landing sequence with the RC transmitter. When the quadrotor has safely landed the safety pilot disables the motors and then powers the quadrotor off.

This methodology is performed and all relevant data is recorded. The quadrotor follows the planned trajectory and replans as necessary to successfully to reach the goal node.

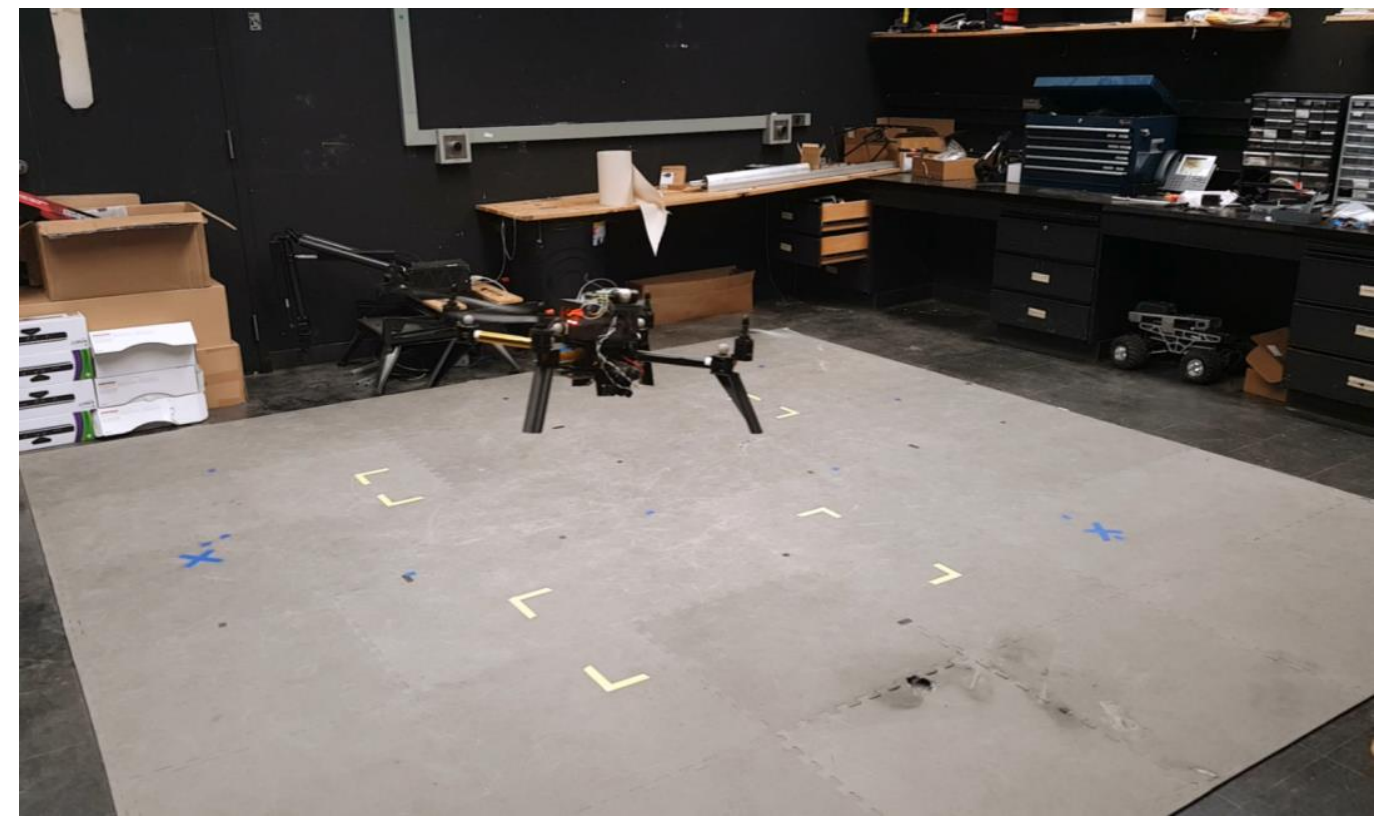

Figure 27: D* Flight Testing

The PRM flight tests were performed using the exact same methodology. The only differences are the Outer Loop control scheme and the Matlab planner, both of which execute PRM instead of $D^{*}$ (see Figure 28 and Figure 29). The same map as well as start and end 
points are used. The control schemes performed correctly and the quadrotor reached the goal destination after it replanned a path around an obstacle. All relevant data is recorded and analyzed in the sections below.

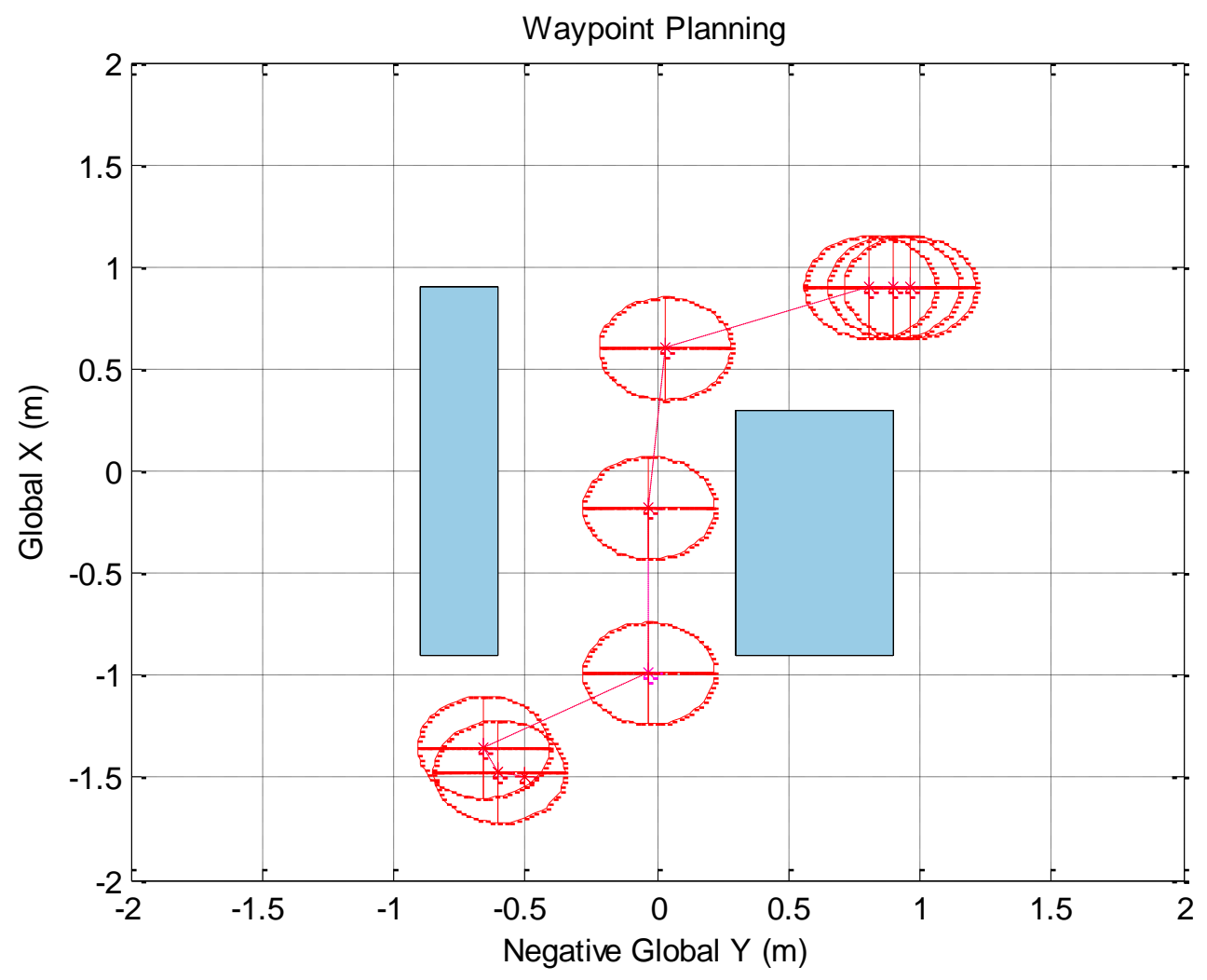

Figure 28: Pre-flight Visualization of map and planned path 


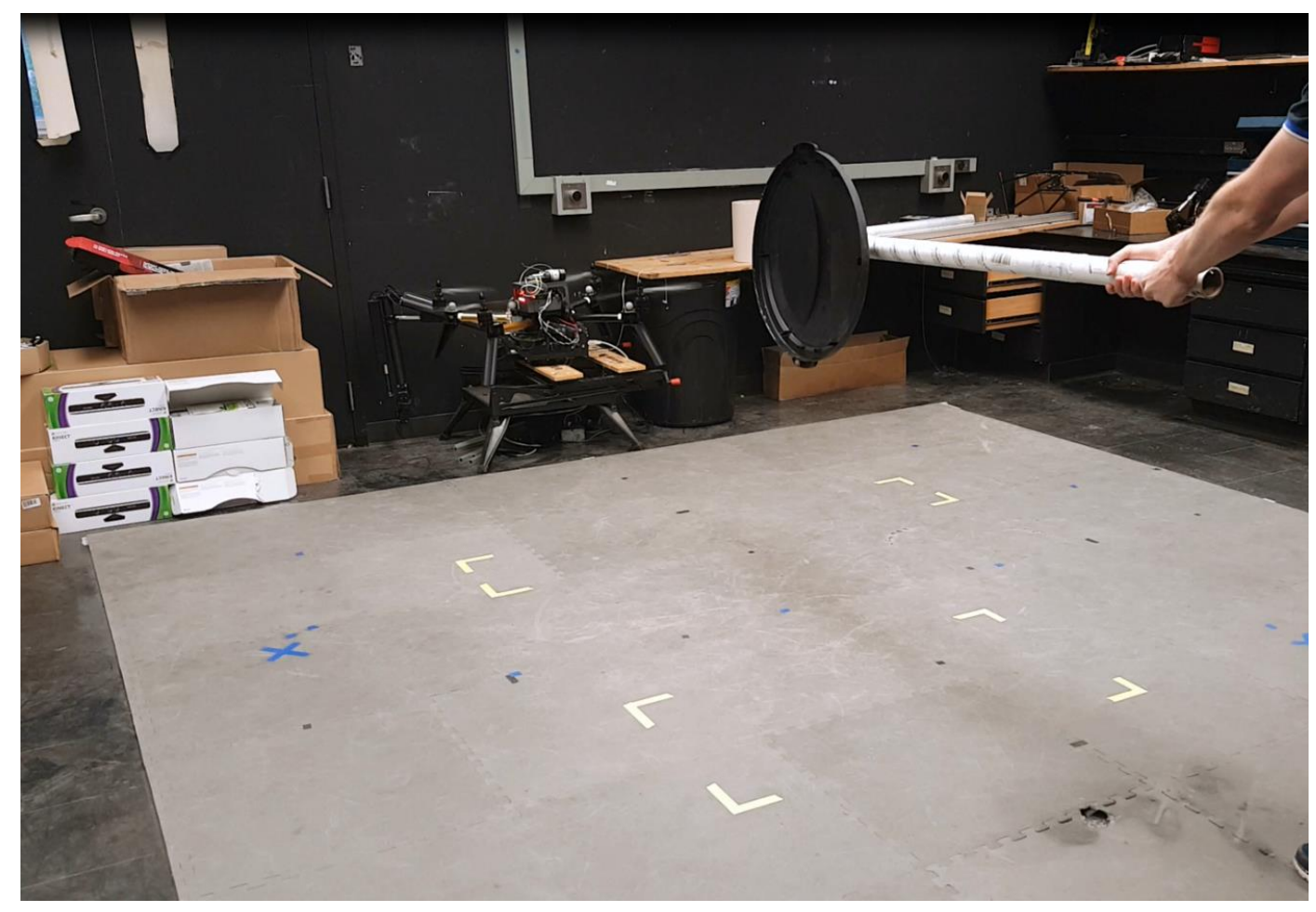

Figure 29: PRM Flight Testing with obstacle in flight path 


\section{Results}

The results of these tests are quite interesting, and a full comparison between $D^{\star}$ and PRM highlights this. The simulations shown in Section IV part A offer straightforward analysis with regards to path length and computational intensity. Path lengths were computed by using the waypoints given by the planner. The distances between each point was calculated and the results are added together and displayed. The results are shown in Table 4 below.

Table 4: Comparison of Path Lengths from Simulation

\begin{tabular}{|c|c|c|c|}
\hline Path \ Method & D* & PRM & Leader (Difference) \\
\hline Original Planned Path & $4.33 \mathrm{~m}$ & $4.66 \mathrm{~m}$ & $\mathrm{D}^{*}(0.33 \mathrm{~m})$ \\
\hline Replanned Path & $5.86 \mathrm{~m}$ & $6.30 \mathrm{~m}$ & $\mathrm{D}^{*}(0.44 \mathrm{~m})$ \\
\hline Combined Length & $8.11 \mathrm{~m}$ & $8.78 \mathrm{~m}$ & $\mathrm{D}^{*}(0.67 \mathrm{~m})$ \\
\hline
\end{tabular}

The Original Planned path is defined as the initial plan of the simulator from the start to the goal node without the unmapped obstacle. The Replanned Path is defined as the path from the crossing point on the unmapped obstacle to the goal node. The Combined Length is defined as the sum of Original Planned Path only up to the point where it meets the obstacle and the Replanned Path. Computational intensity was measured by the time it takes for the computer to execute the simulation in real time. This is achieved using the tic and toc functions built into Matlab, which start a timer and read it, respectively. These tests were all done on the same computer for consistent results. The results are shown below in Table 5. 
Table 5: Comparison of time required from Simulation

\begin{tabular}{|c|c|c|c|}
\hline Time \Method & D* & PRM & Leader (Difference) \\
\hline Original Path Planning & $14.7 \mathrm{sec}$ & $27.2 \mathrm{sec}$ & $D^{*}(12.5 \mathrm{sec})$ \\
\hline Replanning & $10.1 \mathrm{sec}$ & $31.4 \mathrm{sec}$ & $\mathrm{D}^{*}(21.3 \mathrm{sec})$ \\
\hline Total Simulation & $24.8 \mathrm{sec}$ & $53.4 \mathrm{sec}$ & $D^{*}(28.6 \mathrm{sec})$ \\
\hline
\end{tabular}

The Original Path Planning Time is found by placing the tic function at the beginning of the planner and the toc function directly after the path was planned from the start to the goal and waypoints were found, and before any unmapped obstacle is added to the simulation. The Replanning time is calculated by placing a second tic function right after the first toc function, and a second toc function after the planner has found the second set of waypoints. The Total Simulation Time is simply found by adding the two together.

These results are clearly in favor of $\mathrm{D}^{*}$. All distance metrics are shown to be better for the $D^{*}$ simulation, with one third of a meter advantage in planned path distance, and nearly half a meter advantage in replanned distance. The total distance is two thirds of a meter better for the $D^{*}$ planner. This is not a surprise as $D^{*}$ is known for finding an optimal path, while PRM uses the random points, creating a clearly suboptimal path as seen in Figure 24 and Figure 25.

More surprisingly however, are the results from the computational time tests. For just the Originally Planned Path the D* planner takes only approximately $54 \%$ of the time that the PRM planner requires. The Replanning time is even worse, as the $\mathrm{D}^{*}$ planner only needs approximately $39 \%$ of the time that the PRM planner needs. This is most surprising for the Originally Planned Path since, although it calculates a suboptimal path, the PRM planner is quite simple in comparison with the $D^{*}$ algorithm. It is not surprising that $D^{*}$ took less time for the replanning phase since the incremental replan functionality allows the algorithm to only 
modify the arc costs that are affected by the addition of an obstacle to the path instead of a complete replan, as discussed in Section III part D number 1. The PRM method takes a longer amount of time for replanning. This is likely due to the fact that, after adding an obstacle to the graph it must completely replan, this time needing to randomly test more potential nodes since the addition of the obstacle means that there is less space on the graph that is permissible for nodes.

This analysis then begs the question; does PRM actually have any advantages? Another set of tests are performed on a larger grid area using a 1000 by 1000 map, and only the originally planned path. This time the results are much different.

Table 6: Simulation Results for 1000 by 1000 map

\begin{tabular}{|c|c|c|c|}
\hline Time \ Method & D* & PRM & Leader (Difference) \\
\hline Original Planned Path & $25 \mathrm{~min} 11 \mathrm{sec}$ & $31.4 \mathrm{sec}$ & $\begin{array}{c}\text { PRM (24 min } 40 \\
\text { sec) }\end{array}$ \\
\hline
\end{tabular}

As can be seen in Table 6 , the $D^{*}$ method takes a much longer amount of time for the increased resolution map. The PRM method, on the other hand, takes just a little longer than the same method on the 100 by 100 map. To make this a fairer comparison, we can also increase the number of nodes that the PRM method selects to take advantage of the increase in resolution. This is done five times for the original 100 nodes, as well as for 200 nodes and only twice for 300 nodes. The resulting average of each set is shown along with the minimum and maximum values in Table 7. 
Table 7: PRM time comparison for varied number of nodes in 1000 by 1000 map

\begin{tabular}{|c|c|c|c|}
\hline \# of Nodes \Time & PRM Average Time & Max & Min \\
\hline 100 & $30.7 \mathrm{sec}$ & $31.3 \mathrm{sec}$ & $30.3 \mathrm{sec}$ \\
\hline 200 & $7 \mathrm{~min} 9.3 \mathrm{sec}$ & $7 \mathrm{~min} 12.8 \mathrm{sec}$ & $7 \mathrm{~min} 6.7 \mathrm{sec}$ \\
\hline 300 & $36 \min 52.1 \mathrm{sec}$ & $36 \min 55.3 \mathrm{sec}$ & $36 \mathrm{~min} 48.8 \mathrm{sec}$ \\
\hline
\end{tabular}

The trends shown in this data suggest that computation time exponentially increases with a linear increase in the number of nodes. It may be argued that even more nodes may need to be selected for PRM to fully take advantage of the increase in resolution shown in this experiment, however in some situations the number of nodes may not need to increase by a large amount. Therefore, PRM may be a viable method for decreasing computational cost in a large map or high resolution application, so long as the number of nodes selected is within a certain threshold. If this is exceeded, $D^{*}$ may still be the more desirable choice; however it will depend on the scenario.

The testing carried out in Section IV part B shows the actual implementation in a real life, real time flight scenario. The flight path compared to the planned and replanned path for $D^{*}$ is shown in Figure 30 below. The actual flight path is in blue, while the planned and replanned waypoints are shown in red and green, respectively. 


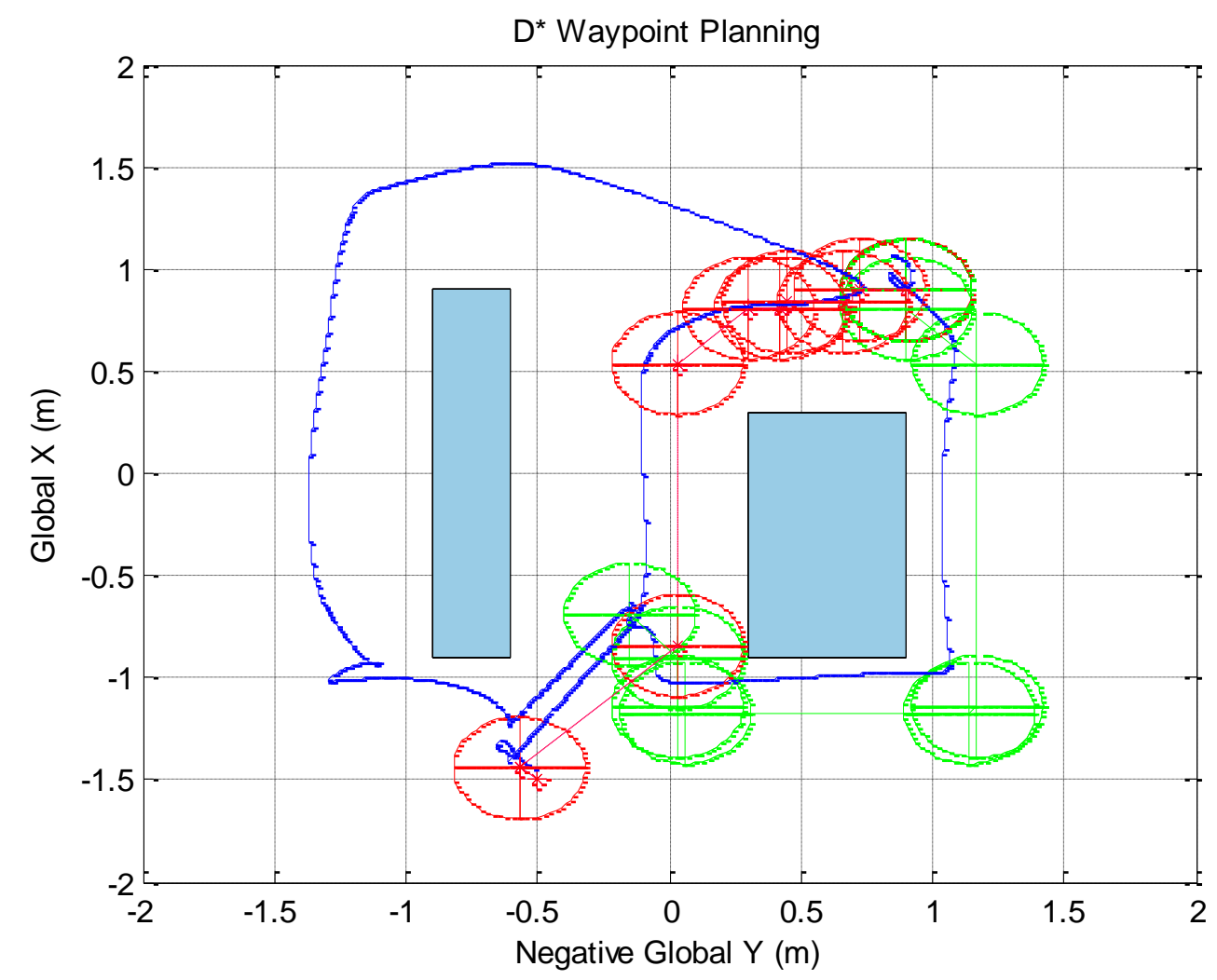

Figure 30: D* Flight Path (in blue) and Replanned Waypoints (in Green)

It can be seen that the quadrotor follows the path quite well for the most part. The only discrepancy is along the bottom right corner of the right obstacle. It is seen here that the quadrotor follows a path along the top left edge of the waypoints cutting very close to the obstacle. The same can be said for the PRM flight path, which follows the top of the waypoints along the bottom edge of the right obstacle, as shown in Figure 31 below. 


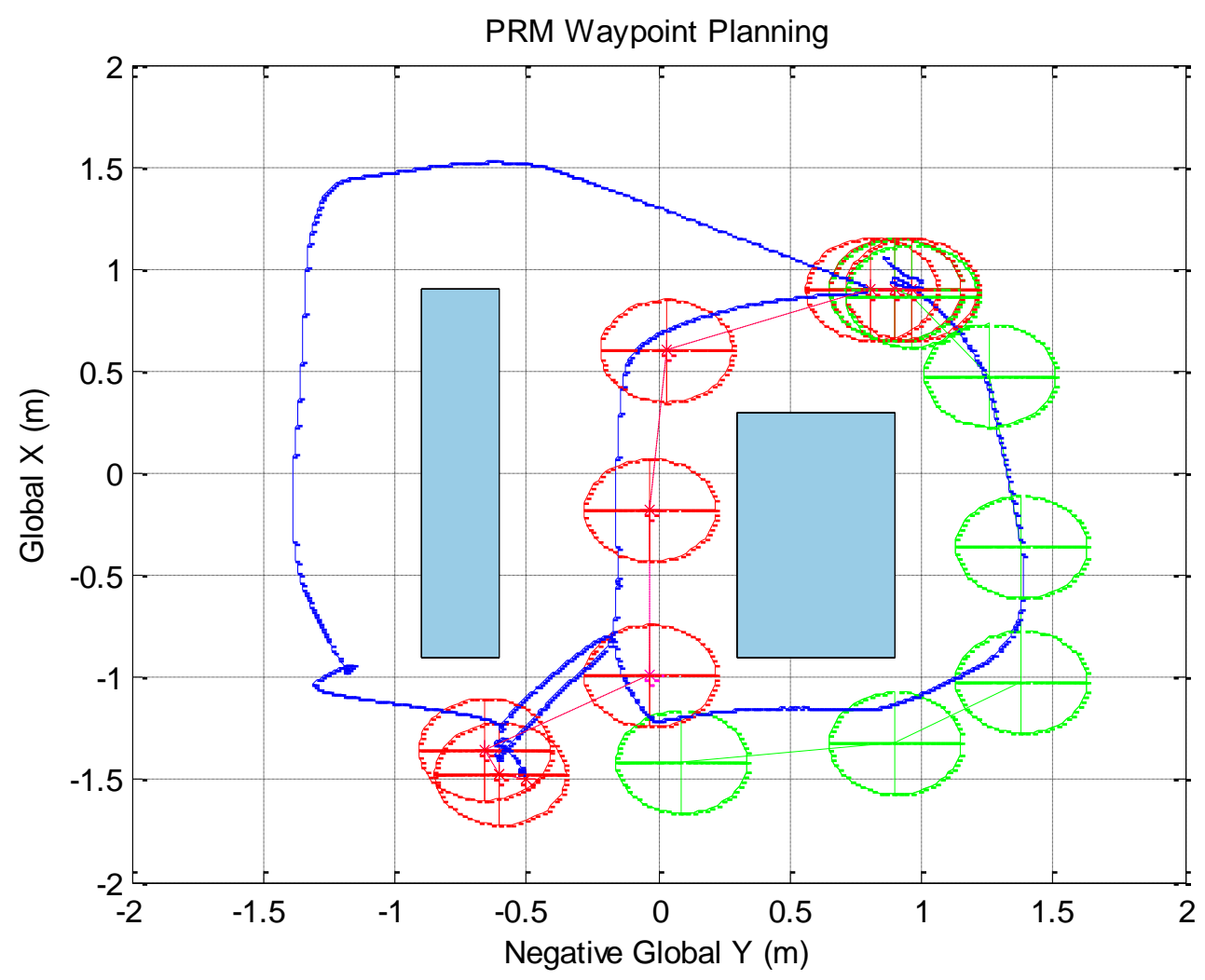

Figure 31: PRM Flight Path (in blue) and Replanned Waypoints (in Green)

This issue is primarily due to the Outer Loop control scheme for the quadrotor, which needs to be tuned in order for the quadrotor to reach the center of these waypoints. A potential fix on the algorithm side would be to increase the size of the buffer zones around the obstacles, but this might make it more difficult for the PRM planner to find a path as this reduces the acceptable area for nodes to be placed and might create some of the narrow passageway issues highlighted in Section III Part D Number 2. Another solution could be to reduce the size of the waypoint spheres to demand more accurate control, but this still may require the Outer Loop control gains to be tuned in order for the quadrotor to reach the smaller spheres. 


\section{Conclusions}

\section{A. Summary}

The problem of path planning from one location to another in a cluttered environment for an aerial robot has been analyzed and discussed at length. Two algorithms, $\mathrm{D}^{*}$ and PRM are chosen to be compared through simulation and testing. The algorithms have been weighed against each other for practical considerations using planning distances and computational speed, as well as complexity and accessibility. A summary of the advantages and disadvantages of each algorithm may be seen in Table 8.

Table 8: Comparison of D* and PRM Planners

\begin{tabular}{|c|c|c|}
\hline & $D^{*}$ & PRM \\
\hline Advantages & 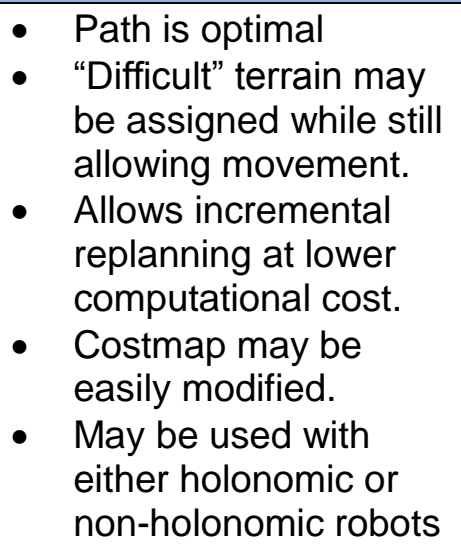 & $\begin{array}{l}\text { - Simple, intuitive } \\
\text { algorithm. } \\
\text { - Can be used in large } \\
\text { mapped environments } \\
\text { where some planners } \\
\text { may not. } \\
\text { - Planning can be done } \\
\text { independently of the } \\
\text { goal location. } \\
\text { Waypoints are easy to } \\
\text { extract. }\end{array}$ \\
\hline Disadvantages & $\begin{array}{l}\text { - } \quad \text { Not as intuitive. } \\
\text { - } \quad \text { Potentially more cost } \\
\text { intensive for larger } \\
\text { areas. } \\
\text { - } \quad \text { Harder to extract } \\
\text { waypoints from path. }\end{array}$ & 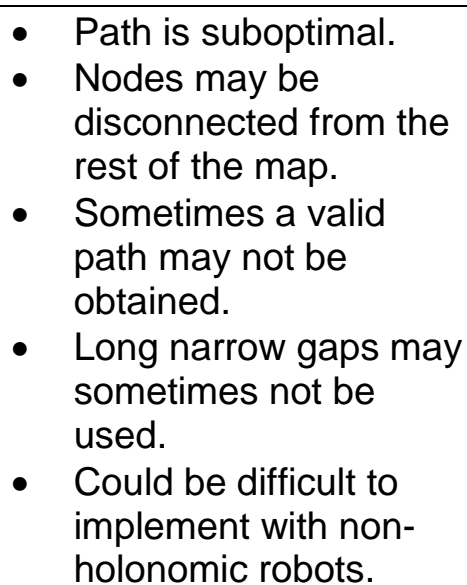 \\
\hline
\end{tabular}

Based on this comparison as well as the analysis from Section $\mathrm{V}$, it may be concluded that $\mathrm{D}^{*}$ is the superior planner given the restrictions that have been discussed. It has an optimal 
path to the goal which may be achieve by holonomic and non-holonomic robots alike, and requires much less computational intensity. It can plan a path through nearly any environment, making it situationally independent. Its only drawbacks might be increased computational cost when scaling to a larger map, waypoints are harder to find, and the algorithm itself is not as straightforward. Although the PRM method easily gives waypoints and is readily understood, it generates a suboptimal path that may not be suitable for non-holonomic robots. It also may not generate a path through narrow passageways unless an additional method is applied. Testing shows that this method also takes more computational power for this application, though if expanded to a larger map PRM might very well be the better choice.

\section{B. Future Work}

In order to achieve outdoor flight, the quadrotor will need an accurate position estimation system to replace the Vicon system. The best answer is to use Global Positioning System (GPS). To get GPS readings, a Novatel OEM-V1 GPS receiver may be interfaced with the quadrotor's avionics board. This has been tested to ensure GPS lock is reliable and consistent. While GPS is a reliable method for outdoor position estimation, its accuracy is generally quite low for an application such as this. Inertial navigation data to determine position could be used and would be highly accurate initially, but the integration process incurs compounding errors which cause drift almost immediately. To remedy this problem, an Extended Kalman Filter (EKF) may be used. An EKF is a recursive nonlinear estimator that can combine measurements using prediction and update phases. In order to restrict the somewhat inaccurate GPS measurements, IMU gyroscope data and accelerometer integration is incorporated for its smoothness and consistency. On the other hand, GPS keeps the accelerometer integration within a certain region over time so that they do not drift too much. This actually allows the best parts of both measurements to be put together to achieve position estimation convergence. An 
Extended Kalman Filter, which has been tested previously on fixed wing autonomous flight, will be used for outdoor position estimation.

For outdoor flights a terrain following method will need to be implemented instead of constant altitude control. This will allow the quadrotor to stay at a consistent altitude going up or down hills or flying over any low obstacles. This has been tested using an ultrasonic rangefinder instead of the laser rangefinder used for object detection, mainly due to the fact that the ultrasonic rangefinder has a detection cone in front of it, where the laser rangefinder only has a narrow beam for detection. Testing has proven this method to be effective indoors, however more testing is necessary before reliable outdoor implementation.

In addition, the PC104 computer will need to be re-implemented into the system to handle Outer Loop control duties for outdoor flights. This will allow flight without a ground station, as discussed earlier, making the quadrotor a much more flexible platform without relying on wireless communication for outdoor control.

Finally, the map used for the planning algorithms will have to be expanded for outdoor applications to allow for larger scale flights. These flights will cover much more area, so a larger map will be necessary while retaining a similar resolution for accurate obstacle avoidance. This will likely increase the processing time needed for the $D^{*}$ algorithm, while the PRM method should not be greatly affected. This has the potential to swing the preferred algorithm in the favor of the PRM method if $D^{*}$ is drastically more computationally intensive. Further experiments and testing will need to verify this. 


\section{References}

[1] Leishman, "The Bréguet-Richet Quad-Rotor Helicopter of 1907," University of Maryland, 2015.

[2] Hirschberg, "The American Helicopter: An Overview of Helicopter Developments in America. 19081999," American Helicopter Society, 2000.

[3] Wannberg, "The Quadrotor Platform from a military point of view," Etteplan, 2012.

[4] Quora, "Why aren't quadrotors used in helicopters rather than the design we see today," Quora, 2014. [Online]. Available: http://www.quora.com/Why-arent-quadrotors-used-in-helicoptersrather-than-the-design-we-see-today. [Accessed 20 June 2015].

[5] "Black Knight Transformer," Advanced Tactics, Inc., 2015. [Online]. Available: https://www.advancedtacticsinc.com/technology/black-knight/. [Accessed 15 June 2015].

[6] "E-volo," E-volo, 2015. [Online]. Available: http://www.e-volo.com/. [Accessed 15 June 2015].

[7] C. Wood, "A closer look at the Malloy Aeronautics Hoverbike," Gizmag, 19 August 2014. [Online]. Available: http://www.gizmag.com/closer-look-malloy-aeronautics-hoverbike/33414/. [Accessed 15 June 2015].

[8] K. Atherton, "The US Army Wants its own Hoverbike, Again," Popular Science, 22 June 2015. [Online]. Available: http://www.popsci.com/pentagon-funding-actual-hoverbike?image=0. [Accessed 25 June 2015].

[9] A. M. Stoll, J. Bevirt, P. P. Pei and E. V. Stilson, "Conceptual Design of the Joby S2 Electric VTOL PAV," in Aviation Technology, Integration, and Operations Conference, Atlanta, Georgia, 2015.

[10] N. E. Serrano, Autonomous Quadrotor Unamnned Aerial Vehicle for Culvert Inspection, Massachusetts Institute of Technology, 2011.

[11] T. K. Venugopalan, T. Taher and G. Barbastathis, "Autonomous Landing of an Unmanned Aerial Vehicle on an Autonomous Marine Vehicle," IEEE, Singapore, 2012.

[12] R. Sampaio, A. Herneandes, M. Becker, F. Catalano, F. Zanini, J. Nobrega and C. Martins, "SquidCop: Design and Evaluation of a Novel Quadrotor MAV for In-flight Launching Air-to-Ground Missions," IEEE, pp. 1-10, 2014.

[13] J. Borenstein, "The HoverBot - An Electrically Powered Flying Robot," Department of Mechanical Engineering and Applied Mechanics, The University of Michigan, Ann Arbor, MI, 1993. 
[14] R. Briand, B. Bluteau and O. Patrouix, "Desing and control of an Outdoor Autonomous Quadrotor powered by a four strokes RC engine," IEEE, Bidart, France, 2006.

[15] E. Cetinsoy, S. Dikyar, C. Hancer, K. T. Oner, E. Sirimoglu, M. Unel and M. Aksit, "Design and construction of a novel quad tilt-wing UAV," Mechatronics, vol. 22, no. 6, p. 723-745, 2012.

[16] R. Huang, Y. Liu and J. J. Zhu, "Guidance, Navigation, and Control System Design for Tripropeller Vertical-Takeoff-and-Landing Unmanned Air Vehicle," Journal of Aircraft, vol. 46, no. 6, pp. 18371856, 2009.

[17] M. Miwa, S. Uemura, Y. Ishihara, A. Imamura, J.-h. Shim and K. Ioi, "Evaluation of quad ducted-fan helicopter," International Journal of Intelligent Unmanned Systems, vol. 1, no. 2, pp. 187-198, 2013.

[18] K. LaFleur, K. Cassady, A. Doud, K. Shades, E. Rogin and B. He, "Quadcopter control in threedimensional spance using a noninvasinve motor imagery-based brain-computer interface," Journal of Neural Engineering, vol. 10, no. 4, 2013.

[19] C. Doyle, J. Bird, T. Isom, J. Kallman, D. Bareiss, D. Dunlop, R. King, J. Abbott and M. Minor, "An Avian-Inspired Passive Mechanism for Quadrotor Perching," IEEE/ASME Transactions on Mechatronics, vol. 18, no. 2, pp. 506-517, 2013.

[20] K. Mohta, V. Kumar and K. Daniilidis, "Vision-based Control of a Quadrotor for Perching on LInes," in IEEE International Conference on Robotics and Automation, Hong Kong, 2014.

[21] J. Yeol and C.-H. Lin, "Development of Multi-Tentacle Micro Air Vehicle," in International Conference on Unmanned Aircraft Systems, Orlando, FL, 2014.

[22] M. J. Cutler, "Design and Control of an Autonomous Variable-Pitch Quadrotor Helicopter," Massachusetts Institute of Technology, Cambridge, Massachusetts, 2012.

[23] M. Cutler, N. K. Ure, B. Michini and J. How, "Comparison of Fixed and Variable Pitch Actuators for Agile Quadrotors," in AIAA Guidance, Navigation, and Control Conference, Minneapolis, MInnesota, 2012.

[24] M. Muller, S. Lupashin and R. D'Andrea, "Quadrocopter Ball Juggling," in IEEE/RSJ International Conference on Intelligent Robots and Systems, San Francisco, CA, USA, 2011.

[25] M. Hehn and R. D'Andrea, "A Flying Inverted Pendulum," in IEEE International Conference on Robotics and Automation, Shanghai, China, 2011.

[26] S. Lupashin, A. Schollig, M. Sherback and R. D'Andrea, "A Simple Learning Strategy for High-Speed Quadrocopter Multi-Flips," in IEEE International Conference on Robotics and Automation, Anchorage, Alaska, USA, 2010. 
[27] E. Davis, B. Nizette and C. Yu, "Development of a Low Cost Quadrotor Platform for Swarm Experiments," in 32nd Chinese Control Conference, Xi'an, 2013.

[28] J. Leonard, A. Savvaris and A. Tsourdos, "Towards a Fully Autonomous Swarm of Unmanned Aerial Vehicles," in UKACC International Conference on Control, Cardiff, UK, 2012.

[29] A. Kushleyev, D. Mellinger and V. Kumar, "Towards A Swarm of Agile Micro Quadrotors," Autonomous Robots, vol. 35, no. 4, pp. 287-300, 2013.

[30] L. Chaimowicz, N. Michael and V. Kumar, "Controlling Swarms of Robots Using Interpolated Implicit Functions," in International Conference on Robotics and Automation, Barcelona, Spain, 2005.

[31] J. How, E. King and Y. Kuwata, "Flight Demonstrations of Cooperative Control for UAV Teams," in AIAA 3rd "Unmanned Unlimited" Technical Conference, Workshop and Exhibit, Chicago, Illinois, 2004.

[32] M. A. Guney and M. Unel, "Formation Control of a Group of Micro Aerial Vehicles (MAVs)," in IEEE International Conference on Systems, Man, and Cybernetics, Manchester, 2013.

[33] Q. Lindsey, D. Mellinger and V. Kumar, "Construction of Cubic Structures with Quadrotor Teams," MIT Press, Cambridge, Massachusetts, 2012.

[34] S. R. B. dos Santos, S. N. Givigi Jr and C. L. N. Junior, "Autonomous Construction of Structures in a Dynamic Environment using Reinforcement Learning," in IEEE International Systems Conference, Orlando, FL, 2013.

[35] M. S. Alvissalim, B. Zaman, A. H. Z., M. A. Ma'sum, G. Jati, W. Jatmiko and P. Mursanto, "Swarm Quadrotor Robots for Telecommunication Network Coverage Area Expansion in Disaster Area," in SICE Annual Conference, Akita, Japan, 2012.

[36] R. Ritz and R. D'Andrea, "Carrying a Flexible Payload with Multiple Flying Vehicles," in IEEE/RSJ International Conference on Intelligent Robots and Systems (IROS), Tokyo, Japan, 2013.

[37] R. Ritz, M. W. Muller, M. Hehn and R. D'Andrea, "Cooperative Quadrocopter Ball Throwing and Catching," in IEEE/RSJ Interational Conference on Intelligent Robots and Systems, Vilamoura, Algarve, Portugal, 2012.

[38] F. Augugliaro, A. P. Schoellig and R. D'Andrea, "Dance of the Flying Machines: Methods for Designing and Executing an Aerial Dance Choreography," IEEE Robotics \& Automation Magazine, vol. 20, no. 4, pp. 96-104, 2013.

[39] M. Waibel, "Controlling a Quadrotor Using Kinect," IEEE Spectrum, 2 July 2011. [Online]. Available: http://spectrum.ieee.org/automaton/robotics/robotics-software/quadrotor-interaction. [Accessed 
21 June 2015].

[40] R. Oung, F. Bourgault, M. Donovan and R. D'Andrea, "The Distributed Flight Array," in IEEE International Conference on Robotics and Automation, Anchorage, Alaska, USA, 2010.

[41] E. W. Dijkstra, "A Note on Two Problems in Connexion with Graphs," Numerische Mathematik, pp. 269-271, 1959.

[42] H. Juzoji, I. Nakajima and T. Kitano, "A Development of Network Topology of Wireless Packet Communications for Disaster Situation with Genetic Algorithms or with Dijkstra's," in IEEE International Conference on Communications, Kyoto, 2011.

[43] M. Parulekar, V. Padte, T. Shah, K. Shroff and R. Shetty, "Automatic Vehicle Navigation using Dijkstra's Algorithm," in ICATE, Mumbai, 2013.

[44] Y. Yi and Y. Guan, "A Path Planning Method to Robot Soccer Based on Dijkstra Algorithm," in Advances in ECWAC, 2012.

[45] P. W. Eklund, S. Kirkby and S. Pollitt, "A Dynamic Multi-source Dijkstra's Algorithm for Vehicle Routing," in Australian New Zealand Conference on Intelligent Information Systems, Adelaide, Australia, 1996.

[46] Z. Yan and Z. Jun, "Dijkstra's Algorithm Based Robus Optimization to Airline Network Planning," in International Conference on Mechanic Automation and Control Engineering, Wuhan, 2010.

[47] L. Verscheure, L. Peyrodie, N. Makni, N. Betrouni, S. Maouche and M. Vermandel, "Dijkstra's Algorithm Applied to 3D Skeletonization of the Brain Vascular Tree: Evaluation and Application to Symbolic," in IEEE EMBS, Buenos Aires, Argentine, 2010.

[48] N. Anastopoulos, K. Nikas, G. Goumas and N. Koziris, "Early Experiences on Accelerating Dijkstra's Algorithm Using Transactional Memory," in IEEE International Symposium on Parallel \& Distributed Processing, Rome, 2009.

[49] N. Jasika, N. Alispahic, A. Elma, K. Ilvana, L. Elma and N. Nosovic, "Dijkstra's shortest path algorithm serial and parallel execution performance analysis," in MIPRO, Opatija, Croatia, 2012.

[50] J.-R. Jiang, H.-W. Huang, J.-H. Liao and S.-Y. Chen, "Extending Dijkstra's Shortest Path Algorithm for Software Defined Networking," in Asia-Pacific Network Operations and Management Symposium, Hsinchu, 2014.

[51] D. Fan and P. Shi, "Improvements of Dijkstra's Algorithm and Its Application in Route Planning," in International Conference on Fuzzy Systems and Knowledge Discovery, Yantai, Shandong, 2010. 
[52] A. Felner, "Position Paper: Dijkstra's Algorithm versus Uniform Cost Search or a Case Against Dijkstra's Algorithm," in Association for the Advancement of Artificial Intelligence, 2011.

[53] P. E. Hart, N. J. Nilsson and B. Raphael, "A Formal Basis for the Heuristic Determination of Minimum Cost Paths," IEEE Transactions of Systems Science and Cybernetics, vol. 4, no. 2, pp. 100-107, 1968.

[54] W. Zeng and R. L. Church, "Finding shortest paths on real road networks: the case for A*," International Journal of Geographical Information Science, vol. 23, no. 4, pp. 531-543, 2009.

[55] J. Yao, C. Lin, X. Xie, A. J. Wang and C.-C. Hung, "Path Planning for Virtual Human Motion Using Improved A* Algorithm," in International Conference on Information Technology, Las Vegas, NV, 2010.

[56] H.-K. Lee, W. Jeong, S. Lee and J. Won, "A Hierarchical Path Planning of cleaning robot Based on Grid Map," in International Conference on Consumer Electronics, Las Vegas, NV, 2013.

[57] M. Ghallab and D. Allard, "Ae - An Efficient Near Admissible Heristic Search Algorithm," in IJCAI, Karlsruhe, Germany, 1983.

[58] H. Wang, J. Zhou, G. Zheng and Y. Liang, "HAS: Hierarchical A-Star algorithm for big map navigation in special areas," in International Conference on Digital Home, Guangzhou, 2014.

[59] A. Stentz, "Optimal and Efficient Path Planning for Partially-Known Environments," in International Conference on Robotics and Automation, 1994.

[60] A. Stentz, "The Focussed D* Algorithm for Real-Time Replanning," in International Joint Conference on Artificial Intelligence, 1995.

[61] S. Koenig and M. Likhachev, "Improved Fast Replanning for Robot Navigation in Unknown Terrain," in International Conference on Robotics \& Automation, Washington, DC, 2002.

[62] D. Ferguson and A. Stentz, "The Delayed D* Algorithm for Efficient Path Replanning," in International Conference on Robotics and Automation, Barcelona, Spain, 2005.

[63] M. Likhachev, D. Ferguson, G. Gordon, A. Stentz and S. Thrun, "Anytime Dynamic A*: An Anytime, Replanning Algorithm," in International Conference on Automated Planning and Scheduling, 2005.

[64] V. Ganapathy, S. C. Yun and T. W. Chien, "Enhanced D* Lite Algorithm for Autonomous Mobile Robot," International Journal of Applied Science and Technology, vol. 1, no. 1, pp. 58-73, 2011.

[65] X. Che, D. Liu and T. Tang, "Path Planning for UCAV in Dynamic and Uncertain Environments Based on Focused D* Algorithm," in International Symposium on Computational Intelligence and Design, Hangzhou, 2011. 
[66] M. Seder, K. Macek and I. Petrovic, "An integrated approach to real-time mobile robot control in partially known indoor environments," in Annual Conference of IEEE Industrial Electronics Society, 2005.

[67] M. Seder and I. Petrovic, "Integration of Focused D* and Witkowski's algorithm for path planning and replanning," in European Conference on Mobile Robots, 2009.

[68] S. Karaman and E. Frazzoli, "Sampling-based Algorithms for Optimal Motion Planning," International Journal of Robotics Research, vol. 30, no. 7, pp. 846-894, 2011.

[69] L. E. Kavraki, P. Svestka, J.-C. Latombe and M. H. Overmars, "Probabilistic Roadmaps for Path Planning in High-Dimensional Configuration Spaces," IEEE Transactions of Robotics and Automation, vol. 12, no. 4, pp. 566-580, 1996.

[70] N. Malone, K. Manavi, J. Wood and L. Tapia, "Construction and Use of Roadmaps That Incorporate Workspace Modeling Errors," in IEEE/RSJ International Conference on Intelligent Robots and Systems, Tokyo, Japan, 2013.

[71] M. Sifyan, H. R. Topcuoglu and M. Ermis, "Path Planning for Mobile Sensor Platforms on a 3-D Terrain Using Hybrid Evolutionary Algorithms," in IEEE Congress on Evolutionary Computation, Barcelona, 2010.

[72] D. Aarno, D. Kragic and H. I. Christensen, "Artificial Potential Biased Probabilistic Roadmap Method," in IEEE International Conference on Robotics and Automation, New Orleans, LA, 2004.

[73] B. Park and W. K. Chung, "Adaptive Node Sampling Method for Probabilistic Roadmap Planners," in International Conference on Intelligent Robots and Systems, St. Louis, USA, 2009.

[74] Z. Jiandong and S. Jianbo, "Narrow Passages Identification for Probabilistic Roadmap Method," in 30th Chinese Control Conference, Yantai, China, 2011.

[75] A. Benitez, D. Vallejo and E. Medrano, "New Technique to Build Probabilistic Roadmap Methods," in 14th International Conference on Electronics, Communications and Computers, 2004.

[76] C. Ekenna, S. A. Jacobs, S. Thomas and N. M. Amato, "Adaptive Neighbor Connection for PRMs: A Natural Fit for Heterogeneous Environments and Parallelism," in IEEE/RSJ International Conference on Intelligent Robots and Systems, Tokyo, Japan, 2013.

[77] Q. Li, C. Wei, J. Wu and X. Zhu, "Improved PRM Method of Low Altitude Penetration Trajectory Planning for UAVs," in IEEE Chinese Guidance, Navigation and Control Conference, Yantai, China, 2014.

[78] G. Wagner, M. Kang and H. Choset, "Probabilistic Path Planning for Multiple Robots with 
Subdimensional Expansion," in IEEE International Conference on Robotics and Automation, Saint Paul, Minnesota, 2012.

[79] J. Yang, P. Dymond and M. Jenkin, "Practicality-Based Probabilistic Roadmaps Method," in Canadian Conference on Computer and Robot Vision, 2011.

[80] N. M. Amato and L. K. Dale, "Probabilistic Roadmap Methods are Embarrassingly Parallel," in International Conference on Robotics and Automation, Detroit, Michigan, 1999.

[81] A. Tayebi and S. McGilvray, "Attitude stabilization of a four-rotor aerial robot," in IEEE Conference on Decision and Control, Atlantis, Paradise Island, Bahamas, 2004.

[82] P. Corke, Robotics, Vision and Control, Berlin Heidelberg: Springer-Verlag, 2011. 